\title{
Entire Domain Basis Function Expansion of the Differential Surface Admittance for Efficient Broadband Characterization of Lossy Interconnects
}

\author{
Martijn Huynen, Member, IEEE, Kamil Yavuz Kapusuz, Student Member, IEEE, Xiao Sun, Geert Van der Plas, \\ Eric Beyne, Daniël De Zutter, Fellow, IEEE, and Dries Vande Ginste, Senior Member, IEEE
}

\begin{abstract}
This paper presents a full-wave method to characterize lossy conductors in an interconnect setting. To this end, a novel and accurate differential surface admittance operator for cuboids based on entire domain basis functions is formulated. By combining this new operator with the augmented electric field integral equation, a comprehensive broadband characterization is obtained. Compared to the state-of-the-art in differential surface admittance operator modeling, we prove the accuracy and improved speed of the novel formulation. Additional examples support these conclusions by comparing results with a commercial software tool and with measurements.
\end{abstract}

Index Terms-3-D differential surface admittance operator, boundary integral equation (BIE), interconnect modeling

\section{INTRODUCTION}

I $\mathrm{N}$ recent years, an unprecedented growth in communication systems and devices has been fueled by the insatiable hunger for information and global connectivity. Video on demand in combination with the ubiquitous use of mobile devices, for example, has led to the advent of 5G, the next generation of mobile communication networks. Another evolution is the inclusion of connectivity functionality in nontraditional devices leading to the internet of things. These emerging technologies and trends do not only reshape communication systems and protocols, but they have repercussions on the hardware level as well. The continuing push for miniaturization and the rising operation frequencies, combined with the integration of circuitry in a wide range of devices and appliances, has led to increasingly intricate and innovative printed circuits boards (PCBs) and integrated circuits (ICs). However, these solutions come at a cost. Particularly, with respect to electromagnetic compatibility and signal and power integrity, effects such as distortion, ringing, skin effect and crosstalk can have detrimental consequences on the correct operation. Hence, for the design of electronics, in particular of the interconnects, electromagnetic solvers are essential tools.

As to the structures they model, electromagnetic solvers have evolved over the years. Pure 2-D structures with a

Manuscript received January 25, 2019. (Corresponding author: M. Huynen) M. Huynen, K. Y. Kapusuz, D. De Zutter and D. Vande Ginste are with the IDLab/Electromagnetics Group, Department of Information Technology, Ghent University/imec B-9052 Ghent, Belgium (email: Martijn.Huynen@UGent.be; KamilYavuz.Kapusuz@UGent.be; Daniel.DeZutter@UGent.be; Dries.VandeGinste@UGent.be).

X. Sun, G. Van der Plas and E. Beyne are with imec, Kapeldreef 75 B-3001 Leuven, Belgium (e-mail: Xiao.Sun@imec.be; Geert.VanderPlas@imec.be; Eric.Beyne@imec.be). fixed cross-section were tackled first [1]-[6]. As the structures shrunk further, the finite length was dealt with by combining 2-D techniques to solve the interior problem, taking the finite conductivity into account, and employing these results to solve the exterior 3-D problem more efficiently [7]-[10]. This did not, however, negate the need for a full-blown threedimensional solver, which has subsequently been researched extensively, also in more general applications [11].

Regardless of the configuration, the available solvers can broadly be classified into two categories. Volumetric formulations, on the one hand, solve the interior problem by meshing the entire volume of the conductors and approximating the fields on this volume mesh. The finite element (FE) method is the prime example [12] of such a method and is widely used in various engineering and scientific fields. Volume integral equations (VIEs) [13]-[15] leverage the Green's function to avoid the use of absorbing boundary conditions to approximate unbounded media, as is required for the FE method, at the cost of a dense system matrix. The family of partial element equivalent circuit (PEEC) methods [7], [8], [16], which is found upon a circuit interpretation of the integral equations, is a popular approach in interconnect modeling. Although these volumetric methods evince great versatility and flexibility in geometry and material modeling, they suffer from a very large number of unknowns due to the considerable increase in mesh elements when the exponential behavior of the skin effect grows stronger for higher frequencies.

Boundary integral equation (BIE) methods, on the other hand, introduce unknowns solely on a surface mesh. This can reduce the system matrix size considerably but at the cost of increased computational complexity. A very general method to simulate homogeneous bodies is the Poggio-Miller-ChanHarrington-Wu-Tsai (PMCHWT) formulation [17]. Nevertheless, the method is poorly suited for the modeling of good conductors as the numerical evaluation of the Green's function in a material with a strongly developed skin effect is an enormous challenge. Even if one employs computationally expensive techniques to bypass this complication [18], [19], the PMCHWT method still has difficulty coping with the high dielectric contrast [20]. Alternative integral formulations, such as N-Müller [21], that do not struggle with high contrast materials, often suffer from accuracy problems - in particular for non-smooth objects [22] — providing a rationale for the prevalence of the PMCHWT formulation.

BIE solvers are a popular choice for interconnect modeling 
as the inclusion of the layered background medium can be achieved through the Green's function [23], [24], as such keeping the number of unknowns under control [25]-[27]. At the same time, the challenge involving the accurate numerical integration of the Green's function in conductors, which plagues the PMCHWT formulation, has to be dealt with as well, given their omnipresence in interconnects. Keeping the discussion restricted to purely surface based methods, methods such as FastImp [28] use singularity cancellation inspired techniques to compute the Green's integrals accurately. In [19], however, it is shown that modifications to singularity cancellation are required to obtain sufficient accuracy for higher frequencies, increasing the complexity and partially negating the efficiency of such methods.

A widely employed alternative approach is to replace the inside material by the background medium and to impose a boundary condition instead; as such avoiding the evaluation of the aforementioned interaction integrals. A popular approximate operator of this type is the (local) surface impedance boundary condition or Leontovich boundary condition [29]. Based on analytical calculations for planar surfaces, this operator is, however, only valid as long as the dielectric contrast and radius of curvature are sufficiently large [30]. Several generalizations to account for these shortcomings have been developed. One method [13] starts from the VIE characterization of the rectangular conductors and by imposing the fields inside to decay exponentially, reduces to an approximate surface formulation. Some approaches approximate each element of the interconnect as an electrically short segment with the current forced along its longitudinal direction and compute a global relation in the cross-section [10], [31], facing integrals involving the 2-D Green's function in conductors. The socalled global impedance boundary condition (GIBC) [32]-[34] invokes the standard 3-D integral equation operators to find a single source integral equation representation but is faced with the same difficulties in the accurate numerical integral evaluation as [28].

The differential surface admittance operator, which introduces a (global) surface relation that takes the material properties exactly into account without intervention of the Green's function, has been introduced as an alternative solution [5]. This operator was first constructed based on the eigenfunctions of a 2-D volume and demonstrated for a rectangle. The method has since been successfully extended to other 2-D shapes [35], [36] and has been applied to the calculation of the parameters for general multiconductor transmission lines [6] and in the analysis of periodic structures [37]. A generalization to arbitrary 2-D shapes based on a contour integral has been presented as well [9] and employed in the context of scattering [38] and interconnect modeling [39], [40] but since it reintroduces the Green's function of the conducting medium it encounters the same problems as the traditional BIE methods.

More recently, the differential surface admittance operator has been extended to 3-D shapes and applied to scattering at cylindrical bodies [41], [42]. Later, we have expanded the 3-D operator to cuboid shapes and employed it to accurately characterize three dimensional interconnects [43]-[45]. In this paper, we revisit the 3-D differential surface admittance operator concept for the cuboid. We formulate a new method based on entire domain basis functions that overcomes one of the main shortcomings of the operator presented in [43] limiting its applicability, namely the need for a large number of eigenmodes and corresponding long calculation time for good conductors. Some preliminary results on this new formulation have been presented before [46], but in this work, the full formalism is derived and detailed, and thoroughly validated and illustrated with meaningful application examples. Afterwards, we combine this improved operator with the augmented electric field integral equation (aEFIE) [47], [48] to obtain a full broadband BIE representation of 3-D interconnects.

In Section II, we present an alternative derivation of the general expression of the 3-D differential surface admittance operator [41], starting from the general theory of resonators and eigenfunctions [49]. A dedicated formulation of the operator's form for a cuboid employing entire domain basis functions is detailed in Section III, where utmost care is taken to exactly include the skin effect by introducing closed analytical expression of infinite sums. Section IV focuses on casting the improved operator into a discrete form compatible with a standard BIE formulation utilizing local basis functions. The discrete operator is then introduced into the augmented EFIE to get a single matrix equation that tackles the entire problem in Section V. Afterwards, in Section VI examples are presented that compare the novel method to the state-of-theart and we also demonstrate the efficiency and appositeness of the operator in modeling interconnects by comparing it with commercial software and measurement results. In Section VII, we formulate our conclusions.

\section{Surface Admittance Operator}

Observe the situation depicted in Fig. 1(a). An arbitrary volume $\mathcal{V}$ with boundary surface $\mathcal{S}$ is composed of a nonmagnetic, homogeneous material, defined by its wavenumber $k$. The time-harmonic fields $\left(e^{j \omega t}\right)$ inside this volume are given by $\left(\mathbf{e}^{\prime}, \mathbf{h}^{\prime}\right)$. The inner region is embedded in a second volume $\mathcal{V}_{0}$ that is filled with a different nonmagnetic, homogeneous material with wavenumber $k_{0}$, also called the background material. Fields in this volume are denoted as $(\mathbf{e}, \mathbf{h})$. Outside $\mathcal{V}_{0}$ arbitrary materials can be present. Impinging source fields $\left(\mathbf{e}_{i}, \mathbf{h}_{i}\right)$ reside anywhere outside $\mathcal{V}$.

In Fig. 1(b), the material inside $\mathcal{V}$ is replaced by that of $\mathcal{V}_{0}$. In order to preserve the fields $(\mathbf{e}, \mathbf{h})$ outside the inner volume, a surface current density $\mathbf{j}_{s}$ is introduced on $\mathcal{S}$. Inside $\mathcal{V}$, the fields are now given by $\left(\mathbf{e}^{\prime \prime}, \mathbf{h}^{\prime \prime}\right)$.

In both situations, the fields inside $\mathcal{V}$ satisfy Maxwell's curl equations

$$
\begin{aligned}
\nabla \times \mathbf{e}^{\prime} & =-j \omega \mu_{0} \mathbf{h}^{\prime} & \nabla \times \mathbf{e}^{\prime \prime} & =-j \omega \mu_{0} \mathbf{h}^{\prime \prime} \\
\nabla \times \mathbf{h}^{\prime} & =j \omega \epsilon \mathbf{e}^{\prime} & \nabla \times \mathbf{h}^{\prime \prime} & =j \omega \epsilon_{0} \mathbf{e}^{\prime \prime}
\end{aligned}
$$

and the relevant boundary conditions

$$
\begin{aligned}
\hat{\mathbf{n}} \cdot \mu_{0}\left(\mathbf{h}-\mathbf{h}^{\prime}\right) & =0 & \hat{\mathbf{n}} \cdot \mu_{0}\left(\mathbf{h}-\mathbf{h}^{\prime \prime}\right) & =0 \\
\hat{\mathbf{n}} \times\left(\mathbf{e}-\mathbf{e}^{\prime}\right) & =0 & \hat{\mathbf{n}} \times\left(\mathbf{e}-\mathbf{e}^{\prime \prime}\right) & =0 \\
\hat{\mathbf{n}} \times\left(\mathbf{h}-\mathbf{h}^{\prime}\right) & =0 & \hat{\mathbf{n}} \times\left(\mathbf{h}-\mathbf{h}^{\prime \prime}\right) & =\mathbf{j}_{s},
\end{aligned}
$$




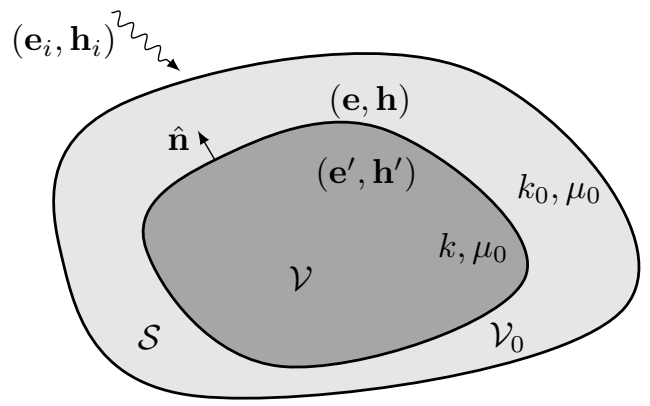

(a) Original situation.

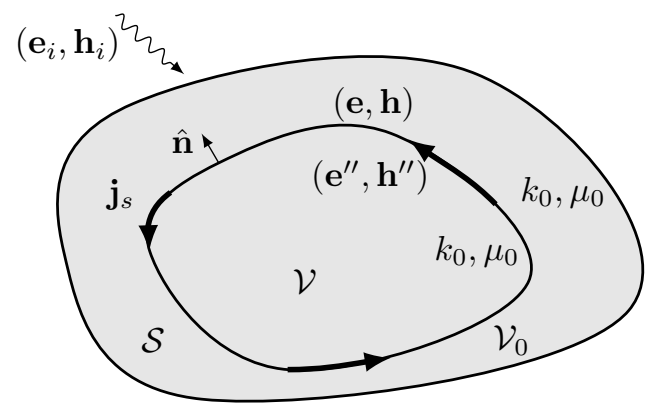

(b) Equivalent situation.

Fig. 1. Problem statement. (a) Arbitrary volume $\mathcal{V}$ with boundary surface $\mathcal{S}$ composed of a homogeneous material with wavenumber $k$. This volume is embedded in another homogeneous medium $\mathcal{V}_{0}$ with wavenumber $k_{0}$. Outside $\mathcal{V}_{0}$ arbitrary materials can be present. In (b), the inner material is replaced by the background medium and a surface current density $\mathbf{j}_{s}$ is introduced on $\mathcal{S}$. All materials are presumed to be nonmagnetic.

where $\hat{\mathbf{n}}$ is the outward-pointing normal on $\mathcal{S}$. Note that the complex permittivity $\epsilon=\epsilon_{r} \epsilon_{0}+\sigma / j \omega$ in (2) includes the conductivity $\sigma$.

By subtracting the relations (1) and (2) that hold for both cases and introducing the field differences as $\mathcal{E}=\mathbf{e}^{\prime}-\mathbf{e}^{\prime \prime}$ and $\mathcal{H}=\mathbf{h}^{\prime}-\mathbf{h}^{\prime \prime}$, we get

$$
\begin{aligned}
\nabla \times \mathcal{E} & =-j \omega \mu_{0} \mathcal{H} \\
\nabla \times \mathcal{H} & =j \omega \epsilon_{0} \mathcal{E}+j \omega\left(\epsilon-\epsilon_{0}\right) \mathbf{e}^{\prime} \equiv j \omega \epsilon_{0} \mathcal{E}+\mathcal{J},
\end{aligned}
$$

where we have designated $j \omega\left(\epsilon-\epsilon_{0}\right) \mathbf{e}^{\prime}$ as an impressed (bulk) electric current $\mathcal{J}$. The same reasoning leads to the following boundary conditions:

$$
\begin{aligned}
\hat{\mathbf{n}} \cdot \mu_{0} \mathcal{H} & =0 \\
\hat{\mathbf{n}} \times \mathcal{E} & =0 \\
\hat{\mathbf{n}} \times \mathcal{H} & =\mathbf{j}_{s} .
\end{aligned}
$$

From (6)-(10) we deduce that the field differences describe the field quantities inside a cavity homogeneously filled with the background material and bounded by perfect electrically conducting walls. It is proved [50] that fields and currents inside this cavity can be expanded into eigenmodes. Generally, these eigenmodes fall apart into two groups: irrotational and divergenceless/solenoidal eigenvectors. Since all fields inside $\mathcal{V}$ for both situations in Fig. 1 are divergence-free, we only need to take the solenoidal eigenmodes into account. As such we can expand $\mathcal{E}, \mathcal{H}$ and $\mathcal{J}$ as

$$
\begin{aligned}
\mathcal{E} & =\sum_{v} a_{v} \mathbf{e}_{v} \\
\mathcal{H} & =\sum_{v} b_{v} \mathbf{h}_{v} \\
\mathcal{J} & =\sum_{v} c_{v} \mathbf{e}_{v},
\end{aligned}
$$

with $v$ a triple index and $\mathbf{e}_{v}$ and $\mathbf{h}_{v}$ the electric and magnetic solenoidal eigenmodes of $\mathcal{V}$, respectively. The curls of the field differences are expanded, separately, as well:

$$
\begin{aligned}
\nabla \times \mathcal{E} & =\sum_{v} r_{v} \mathbf{h}_{v} \\
\nabla \times \mathcal{H} & =\sum_{v} s_{v} \mathbf{e}_{v} .
\end{aligned}
$$

Plugging these expansions into (6) and (7) and taking the orthogonality of the eigenmodes into account, the following relations between the expansion coefficients are found:

$$
\begin{aligned}
& r_{v}=-j \omega \mu_{0} b_{v} \\
& s_{v}=j \omega \epsilon_{0} a_{v}+c_{v} .
\end{aligned}
$$

In determining the remaining coefficients, we will project (13)-(15) onto the various eigenvectors and integrate over $\mathcal{V}$. For this, we will exploit a few properties of these eigenvectors [49]. Note in particular that the eigenmodes can be chosen to be real. Moreover, we require the eigenmodes to be normalized such that $k_{v} \mathbf{e}_{v}=\nabla \times \mathbf{h}_{v}$ and $k_{v} \mathbf{h}_{v}=\nabla \times \mathbf{e}_{v}$ with $k_{v}$ the shared wavenumber of $\mathbf{e}_{v}$ and $\mathbf{h}_{v}$. A consequence of this is that both sets of eigenmodes share the same normalization constant $\mathcal{N}_{v}$ :

$$
\mathcal{N}_{v}^{2}=\int_{\mathcal{V}}\left|\mathbf{e}_{v}\right|^{2} \mathrm{~d} V=\int_{\mathcal{V}}\left|\mathbf{h}_{v}\right|^{2} \mathrm{~d} V .
$$

We start by projecting both sides of (13) onto $\mathbf{e}_{w}$ and invoking the eigenmode orthogonality, which leads to

$$
c_{w} \mathcal{N}_{w}^{2}=\int_{\mathcal{V}} \mathcal{J} \cdot \mathbf{e}_{w} \mathrm{~d} V=j \omega\left(\epsilon-\epsilon_{0}\right) \int_{\mathcal{V}} \mathbf{e}_{w} \cdot \mathbf{e}^{\prime} \mathrm{d} V .
$$

By utilizing the Helmholtz equation for $\mathbf{e}_{w}$ and the appropriate integral relationships, the integral on the right-hand side is rewritten as

$$
\begin{aligned}
& k_{w}^{2} \int_{\mathcal{V}} \mathbf{e}_{w} \cdot \mathbf{e}^{\prime} \mathrm{d} V=\int_{\mathcal{V}} \mathbf{e}_{w} \cdot\left(\nabla \times \nabla \times \mathbf{e}^{\prime}\right) \mathrm{d} V \\
& +\int_{\mathcal{S}}\left(\hat{\mathbf{n}} \times \mathbf{e}_{w}\right) \cdot\left(\nabla \times \mathbf{e}^{\prime}\right) \mathrm{d} S-\int_{\mathcal{S}}\left(\hat{\mathbf{n}} \times \mathbf{e}^{\prime}\right) \cdot\left(\nabla \times \mathbf{e}_{w}\right) \mathrm{d} S .
\end{aligned}
$$

Subsequently, the first integral on the right-hand side is transformed to the sought-after integral by invoking the Helmholtz equation for $\mathbf{e}^{\prime}$ while the penultimate term drops out as the tangential component of $\mathbf{e}_{w}$ vanishes on $\mathcal{S}$. This leads to

$$
\left(k^{2}-k_{w}^{2}\right) \int_{\mathcal{V}} \mathbf{e}_{w} \cdot \mathbf{e}^{\prime} \mathrm{d} V=k_{w} \int_{\mathcal{S}}\left(\hat{\mathbf{n}} \times \mathbf{e}^{\prime}\right) \cdot \mathbf{h}_{w} \mathrm{~d} S .
$$


Hence, we find the following expression for $c_{w}$ :

$$
c_{w}=\frac{\left(k_{0}^{2}-k^{2}\right) k_{w}}{j \omega \mu_{0}\left(k^{2}-k_{w}^{2}\right) \mathcal{N}_{w}^{2}} \int_{\mathcal{S}}\left(\hat{\mathbf{n}} \times \mathbf{e}^{\prime}\right) \cdot \mathbf{h}_{w} \mathrm{~d} S .
$$

Using the same approach for (14) but projecting on $\mathbf{h}_{w}$ and using the adequate vector property leads to

$$
r_{w} \mathcal{N}_{w}^{2}=\int_{\mathcal{V}} \nabla \cdot\left(\mathcal{E} \times \mathbf{h}_{w}\right) \mathrm{d} V+\int_{\mathcal{V}} \mathcal{E} \cdot\left(\nabla \times \mathbf{h}_{w}\right) \mathrm{d} V .
$$

The first integral can be transformed to a surface integral by the divergence theorem and promptly vanishes because of (9). For the second integral we substitute the expansion for $\mathcal{E}$ :

$$
r_{w} \mathcal{N}_{w}^{2}=k_{w} \sum_{v} a_{v} \int_{\mathcal{V}} \mathbf{e}_{v} \cdot \mathbf{e}_{w} \mathrm{~d} V
$$

By once again invoking the mode orthogonality, we get the following simple relation between $r_{w}$ and $a_{w}$ :

$$
r_{w}=k_{w} a_{w} .
$$

Starting from (15), an analogous reasoning leads to the following expression for $s_{w}$ :

$$
s_{w}=k_{w} b_{w} .
$$

Combining (22), (25) and (26) with (16)-(17) enables us to find the following expressions for $\mathcal{E}$ and $\mathcal{H}$ :

$$
\begin{aligned}
\mathcal{E} & =\sum_{v} \mathbf{e}_{v} \frac{-j \omega \mu_{0} \eta k_{v}}{\left(k_{0}^{2}-k_{v}^{2}\right)\left(k^{2}-k_{v}^{2}\right) \mathcal{N}_{v}^{2}} \int_{\mathcal{S}}\left(\hat{\mathbf{n}} \times \mathbf{e}^{\prime}\right) \cdot \mathbf{h}_{v} \mathrm{~d} S \\
\mathcal{H} & =\sum_{v} \mathbf{h}_{v} \frac{\eta k_{v}^{2}}{\left(k_{0}^{2}-k_{v}^{2}\right)\left(k^{2}-k_{v}^{2}\right) \mathcal{N}_{v}^{2}} \int_{\mathcal{S}}\left(\hat{\mathbf{n}} \times \mathbf{e}^{\prime}\right) \cdot \mathbf{h}_{v} \mathrm{~d} S,
\end{aligned}
$$

with the contrast parameter $\eta=\left(k^{2}-k_{0}^{2}\right) / j \omega \mu_{0}$.

Plugging (28) into (10) and employing (4), i.e., $\hat{\mathbf{n}} \times \mathbf{e}^{\prime}=$ $\hat{\mathbf{n}} \times \mathbf{e}$, gives us the following relation between $\mathbf{j}_{s}$ and the tangential electric field $\mathbf{e}_{t}$ on $\mathcal{S}$ :

$$
\mathbf{j}_{s}=-\eta \sum_{v} \frac{\mathcal{K}_{v}}{\mathcal{N}_{v}^{2}}\left[\int_{\mathcal{S}}\left(\hat{\mathbf{n}} \times \mathbf{h}_{v}\right) \cdot \mathbf{e}_{t} \mathrm{~d} S\right]\left(\hat{\mathbf{n}} \times \mathbf{h}_{v}\right),
$$

where $\mathcal{K}_{v}=k_{v}^{2} /\left(k_{0}^{2}-k_{v}^{2}\right) /\left(k^{2}-k_{v}^{2}\right)$. This relation can be cast as an operator $\mathbf{j}_{s}=\mathcal{Y} \mathbf{e}_{t}$, i.e., a 3-D differential surface admittance operator.

\section{CONTINuOUS $\mathcal{Y}$-OPERATOR FOR A CUBOID}

The 3-D differential surface admittance operator has been employed before for cylindrical structures [41] and cuboids [43], albeit introduced from a different perspective. Here, we will thoroughly revisit the relevant case of the cuboid and present an improved approach to calculate the $\mathcal{Y}$-operator that is not only more compact but also much better suited for accurate numerical calculation. In particular, the novel approach deals with the skin effect in a much more efficient way.

\section{A. Expansion matrix}

The starting point are the divergenceless magnetic eigenmodes $\mathbf{h}_{m}$ for a cuboid with dimensions $\left\{l_{x}, l_{y}, l_{z}\right\}$. These eigenmodes fall apart in two types: transverse electric (TE) and transverse magnetic (TM) modes, where transverse is defined with respect to the $z$-axis. The wavenumbers of both sets of modes are given by

$k_{\nu}^{2}=k_{m n p}^{2}=\left(\frac{m \pi}{l_{x}}\right)^{2}+\left(\frac{n \pi}{l_{y}}\right)^{2}+\left(\frac{p \pi}{l_{z}}\right)^{2}=\lambda_{x}^{2}+\lambda_{y}^{2}+\lambda_{z}^{2}$,

with $\nu=(m, n, p)$ the triple index defined over the nonnegative integers excluding $m=n=p=0$.

The magnetic TE eigenmodes are defined as

$$
\begin{aligned}
\mathbf{h}_{m n p}^{\mathrm{TE}} & =\lambda_{z} \lambda_{x} \sin \left(\lambda_{x} x\right) \cos \left(\lambda_{y} y\right) \cos \left(\lambda_{z} z\right) \hat{\mathbf{x}} \\
& +\lambda_{z} \lambda_{y} \cos \left(\lambda_{x} x\right) \sin \left(\lambda_{y} y\right) \cos \left(\lambda_{z} z\right) \hat{\mathbf{y}} \\
& -\left(\lambda_{x}^{2}+\lambda_{y}^{2}\right) \cos \left(\lambda_{x} x\right) \cos \left(\lambda_{y} y\right) \sin \left(\lambda_{z} z\right) \hat{\mathbf{z}},
\end{aligned}
$$

while the magnetic TM modes are expressed as

$$
\begin{aligned}
\mathbf{h}_{m n p}^{\mathrm{TM}} & =k_{m n p} \lambda_{y} \sin \left(\lambda_{x} x\right) \cos \left(\lambda_{y} y\right) \cos \left(\lambda_{z} z\right) \hat{\mathbf{x}} \\
& -k_{m n p} \lambda_{x} \cos \left(\lambda_{x} x\right) \sin \left(\lambda_{y} y\right) \cos \left(\lambda_{z} z\right) \hat{\mathbf{y}} .
\end{aligned}
$$

For $p=0$ or $m=0=n, \mathbf{h}_{m n p}^{\mathrm{TE}}$ is zero while $\mathbf{h}_{m n p}^{\mathrm{TM}}$ vanishes for $m=0$ or $n=0$.

Both types of eigenvectors have closed expressions for their normalization constants $\mathcal{N}_{m n p}^{2}$ :

$$
\begin{aligned}
\mathrm{TE}: \mathcal{N}_{m n p}^{2} & =k_{m n p}^{2} \frac{V}{2}\left(\lambda_{x}^{2}+\lambda_{y}^{2}\right) \frac{1}{\varepsilon_{m} \varepsilon_{n}}, \\
\mathrm{TM}: \mathcal{N}_{m n p}^{2} & =k_{m n p}^{2} \frac{V}{4}\left(\lambda_{x}^{2}+\lambda_{y}^{2}\right) \frac{1}{\varepsilon_{p}},
\end{aligned}
$$

with $V=l_{x} l_{y} l_{z}$, i.e., the volume of $\mathcal{V}$, and $\varepsilon_{i}$ the Neumann factor [49], which equals 1 for $i=0$ and 2 otherwise.

In (29), the eigenmodes themselves are actually not required but rather their tangential component on $\mathcal{S}$. Numbering the six faces $S_{i}$ from 0 to 5 , corresponding to the outward-pointing normal vectors $(-\hat{\mathbf{x}}, \hat{\mathbf{x}},-\hat{\mathbf{y}}, \hat{\mathbf{y}},-\hat{\mathbf{z}}, \hat{\mathbf{z}})$, they are located at $x=$ $0, x=l_{x}, y=0, y=l_{y}, z=0, z=l_{z}$, respectively.

The rotated magnetic eigenmodes on $S_{0}$ are thus given by

$$
\begin{aligned}
S_{0}:-\hat{\mathbf{x}} \times \mathbf{h}_{m n p}= & -\zeta_{y, m n p} \sin \left(\lambda_{y} y\right) \cos \left(\lambda_{z} z\right) \hat{\mathbf{z}} \\
& +\zeta_{z, m n p} \sin \left(\lambda_{z} z\right) \cos \left(\lambda_{y} y\right) \hat{\mathbf{y}},
\end{aligned}
$$

where we have introduced $\zeta_{\beta, m n p}$ as a placeholder for the pertinent factor of the $\beta$-component of either TE or TM modes, where $\beta$ stands for $x, y$ or $z$, and made explicitly clear that they (can) depend on all three indices. Remark that $\zeta_{z, m n p}$ equals zero for TM modes. Careful inspection of the same quantity on the other sides of the cuboid shows that they all have the same form: each is composed of two terms along the two axes which comprise the face and, in turn, each term contains the product of a sine and cosine function. Furthermore, it turns out that the contribution along a particular axis shows a cosine dependence for the coordinate along that axis and a sine dependence along the remaining axis. In (35), for example, the first term oriented along $\hat{\mathbf{z}}$ has a $\cos \left(\lambda_{z} z\right)$ and a $\sin \left(\lambda_{y} y\right)$ dependence.

This observation prompts us to expand both $\mathbf{e}_{t}$ and $\mathbf{j}_{s}$ in similar functions, i.e., entire domain basis functions, on 
each face and exploit the orthogonality of the trigonometric functions. For example, the tangential electric field on $S_{0}$ is expanded as

$$
\begin{aligned}
S_{0}: \mathbf{e}_{t}=\sum_{n^{\prime}, p^{\prime}} \mathbf{e}_{0, n^{\prime} p^{\prime}} & =\sum_{n^{\prime}, p^{\prime}}\left(a_{0, n^{\prime} p^{\prime}}^{0} \sin \left(\lambda_{y}^{\prime} y\right) \cos \left(\lambda_{z}^{\prime} z\right) \hat{\mathbf{z}}\right. \\
& \left.+a_{0, n^{\prime} p^{\prime}}^{1} \sin \left(\lambda_{z}^{\prime} z\right) \cos \left(\lambda_{y}^{\prime} y\right) \hat{\mathbf{y}}\right), \quad
\end{aligned}
$$

with $n^{\prime}$ and $p^{\prime}$ being the two indices governing the expansion along the $y$ - and $z$-axis, respectively, $\lambda_{y}^{\prime}$ and $\lambda_{z}^{\prime}$ fulfilling the same role as in (30) but for these primed indices $n^{\prime}$ and $p^{\prime}$, and $a_{0, n^{\prime} p^{\prime}}^{0}$ and $a_{0, n^{\prime} p^{\prime}}^{1}$ the unknown expansion coefficients on $S_{0}$ along its two axes.

For every term $\mathbf{e}_{0, n^{\prime} p^{\prime}}$, the integral in (29) can then be expressed as

$$
\begin{aligned}
& \int_{S_{0}}\left(-\hat{\mathbf{x}} \times \mathbf{h}_{m n p}\right) \cdot \mathbf{e}_{0, n^{\prime} p^{\prime}} \mathrm{d} S \\
& =-\delta_{n n^{\prime}} \delta_{p p^{\prime}} \frac{A_{0}}{2}\left[a_{0, n^{\prime} p^{\prime}}^{0} \zeta_{y, m n p} \frac{\sigma_{n}}{\varepsilon_{p}}-a_{0, n^{\prime} p^{\prime}}^{1} \zeta_{z, m n p} \frac{\sigma_{p}}{\varepsilon_{n}}\right]
\end{aligned}
$$

with $A_{0}$ being the area of face $S_{0}, \sigma_{i}$ is zero for $i=0$ and 1 otherwise, and $\delta_{i j}$ being the Kronecker delta. These Kronecker deltas imply that we can henceforth replace $\left\{n^{\prime}, p^{\prime}\right\}$ by $\{n, p\}$. Focusing now, for illustration purposes, on the expansions coefficients $a_{0, n p}^{0}$ along $\hat{\mathbf{z}}$ and plugging the results back into (29) gives

$$
\mathbf{j}_{s}=\frac{\eta A_{0}}{2} \sum_{n, p} \frac{\sigma_{n}}{\epsilon_{p}} \sum_{m} \frac{\mathcal{K}_{m n p}}{\mathcal{N}_{m n p}^{2}} a_{0, n p}^{0} \zeta_{y, m n p}\left(\hat{\mathbf{n}} \times \mathbf{h}_{m n p}\right) .
$$

At this point, we can expand the surface current density on each face in exactly the same way as the tangential electric field. Illustrating this for $S_{0}$, we get

$$
\begin{aligned}
S_{0}: \mathbf{j}_{s}=\sum_{n^{\prime \prime}, p^{\prime \prime}} \mathbf{j}_{0, n^{\prime \prime} p^{\prime \prime}} & =\sum_{n^{\prime \prime}, p^{\prime \prime}}\left(b_{0, n^{\prime \prime} p^{\prime \prime}}^{0} \sin \left(\lambda_{y}^{\prime \prime} y\right) \cos \left(\lambda_{z}^{\prime \prime} z\right) \hat{\mathbf{z}}\right. \\
& \left.+b_{0, n^{\prime \prime} p^{\prime \prime}}^{1} \sin \left(\lambda_{z}^{\prime \prime} z\right) \cos \left(\lambda_{y}^{\prime \prime} y\right) \hat{\mathbf{y}}\right), \quad(39)
\end{aligned}
$$

where we have introduced the double primed indices $n^{\prime \prime}$ and $p^{\prime \prime}$ analogously to the primed ones in (36). Focusing again on a single component (along the $z$-axis) and introducing the relevant term of $\hat{\mathbf{n}} \times \mathbf{h}_{m n p}$ on the same face, (38) yields

$$
\begin{aligned}
& b_{0, n^{\prime \prime} p^{\prime \prime}}^{0} \sin \left(\lambda_{y}^{\prime \prime} y\right) \cos \left(\lambda_{z}^{\prime \prime} z\right)= \\
& \frac{-\eta A_{0}}{2} \sum_{n, p} \frac{\sigma_{n}}{\epsilon_{p}} \sum_{m} \frac{\mathcal{K}_{m n p}}{\mathcal{N}_{m n p}^{2}} a_{0, n p}^{0} \zeta_{y, m n p}^{2} \sin \left(\lambda_{y} y\right) \cos \left(\lambda_{z} z\right) .
\end{aligned}
$$

In order to isolate $b_{0, n^{\prime \prime} p^{\prime \prime}}^{0}$, we multiply both sides by $\sin \left(\lambda_{y}^{\prime \prime} y\right) \cos \left(\lambda_{z}^{\prime \prime} z\right)$ and integrate over $S_{0}$. This once again forces the (double) primed indices to take the same values as their nonprimed counterparts, leading to

$$
b_{0, n p}^{0}=\frac{A_{0} \sigma_{n}}{2 \epsilon_{p}}\left[-\eta \sum_{m} \frac{\mathcal{K}_{m n p}}{\mathcal{N}_{m n p}^{2}} \zeta_{y, m n p}^{2}\right] a_{0, n p}^{0} .
$$

This equation fully captures the influence of a single $a_{0, n p}^{0}$ component of the tangential electric field on its counterpart, i.e., the $b_{0, n p}^{0}$ component, of the surface current density. For the $y$-component of $\mathbf{j}_{s}$ in (39), we find a similar relation:

$$
b_{0, n p}^{1}=\frac{A_{0} \sigma_{n}}{2 \epsilon_{p}}\left[\eta \sum_{m} \frac{\mathcal{K}_{m n p}}{\mathcal{N}_{m n p}^{2}} \zeta_{y, m n p} \zeta_{z, m n p}\right] a_{0, n p}^{0} .
$$

The same procedure can now be repeated for the other five faces to fully determine the impact that this one component of the expanded tangential electric field has on the surface current densities on all faces. We will pick a few of these components to showcase their most important characteristics.

First, a closer look at the opposite face, i.e., $S_{1}$, shows that the rotated magnetic eigenmode and surface current density are given by

$$
\begin{aligned}
S_{1}: \hat{\mathbf{x}} \times \mathbf{h}_{m n p}= & +\zeta_{y, m n p}(-1)^{m} \sin \left(\lambda_{y} y\right) \cos \left(\lambda_{z} z\right) \hat{\mathbf{z}} \\
& -\zeta_{z, m n p}(-1)^{m} \sin \left(\lambda_{z} z\right) \cos \left(\lambda_{y} y\right) \hat{\mathbf{y}},
\end{aligned}
$$

$$
\begin{aligned}
S_{1}: \mathbf{j}_{s}=\sum_{n^{\prime \prime}, p^{\prime \prime}} \mathbf{j}_{1, n^{\prime \prime} p^{\prime \prime}} & =\sum_{n^{\prime \prime}, p^{\prime \prime}} b_{1, n^{\prime \prime} p^{\prime \prime}}^{0} \sin \left(\lambda_{y}^{\prime \prime} y\right) \cos \left(\lambda_{z}^{\prime \prime} z\right) \hat{\mathbf{z}} \\
& +b_{1, n^{\prime \prime} p^{\prime \prime}}^{1} \sin \left(\lambda_{z}^{\prime \prime} z\right) \cos \left(\lambda_{y}^{\prime \prime} y\right) \hat{\mathbf{y}} .
\end{aligned}
$$

Following the same reasoning as outlined above, we thus find the following relation between $a_{0, n p}^{0}$ and $b_{1, n p}^{0}$ :

$$
b_{1, n p}^{0}=\frac{A_{0} \sigma_{n}}{2 \epsilon_{p}} s\left[\eta \sum_{m} \frac{\mathcal{K}_{m n p}}{\mathcal{N}_{m n p}^{2}}(-1)^{m} \zeta_{y, m n p}^{2}\right] a_{0, n p}^{0},
$$

while for $b_{1, n p}^{1}$ we get

$$
b_{1, n p}^{1}=\frac{A_{0} \sigma_{n}}{2 \epsilon_{p}}\left[-\eta \sum_{m} \frac{\mathcal{K}_{m n p}}{\mathcal{N}_{m n p}^{2}}(-1)^{m} \zeta_{y, m n p} \zeta_{z, m n p}\right] a_{0, n p}^{0} .
$$

We immediately see that (45) and (46) are very similar to (41) and (42), respectively; the only difference is a minus sign and the factor $(-1)^{m}$ in the summation.

Next, we observe the influence on a perpendicular face, e.g., $S_{2}$. Here, the rotated magnetic eigenmodes and the expansion of $\mathbf{j}_{s}$ are given by

$$
\begin{aligned}
S_{2}:-\hat{\mathbf{y}} \times \mathbf{h}_{m n p}= & -\zeta_{z, m n p} \sin \left(\lambda_{z} z\right) \cos \left(\lambda_{x} x\right) \hat{\mathbf{x}} \\
& +\zeta_{x, m n p} \sin \left(\lambda_{x} x\right) \cos \left(\lambda_{z} z\right) \hat{\mathbf{z}}
\end{aligned}
$$

$$
\begin{aligned}
S_{2}: \mathbf{j}_{s}=\sum_{p^{\prime \prime}, m^{\prime \prime}} \mathbf{j}_{2, p^{\prime \prime} m^{\prime \prime}} & =\sum_{p^{\prime \prime}, m^{\prime \prime}} b_{2, p^{\prime \prime} m^{\prime \prime}}^{0} \sin \left(\lambda_{z}^{\prime \prime} z\right) \cos \left(\lambda_{x}^{\prime \prime} x\right) \hat{\mathbf{x}} \\
& +b_{2, p^{\prime \prime} m^{\prime \prime}}^{1} \sin \left(\lambda_{x}^{\prime \prime} x\right) \cos \left(\lambda_{z}^{\prime \prime} z\right) \hat{\mathbf{z}} . \quad
\end{aligned}
$$

Substituting these in (38) and selecting the component along the $x$-axis, we obtain the equivalent of (40) as

$$
\begin{aligned}
& b_{2, p^{\prime \prime} m^{\prime \prime}}^{0} \sin \left(\lambda_{z}^{\prime \prime} z\right) \cos \left(\lambda_{x}^{\prime \prime} x\right)= \\
& \frac{-\eta A_{0}}{2} \sum_{n, p} \frac{\sigma_{n}}{\epsilon_{p}} \sum_{m} \frac{\mathcal{K}_{m n p}}{\mathcal{N}_{m n p}^{2}} a_{0, n p}^{0} \zeta_{y, m n p} \zeta_{z, m n p} \sin \left(\lambda_{z} z\right) \cos \left(\lambda_{x} x\right) .
\end{aligned}
$$

Employing a similar projection to isolate $b_{2, p^{\prime \prime} m^{\prime \prime}}^{0}$ as before, the double primed indices collapse onto their nonprimed equivalents. This time, however, these indices, i.e., $m$ and $p$, do not correspond (completely) with the indices of $a_{0, n p}^{0}$, i.e., $n$ and $p$. As such, this projection enforces an extra condition on the index $m$ and the summation, as found for example in (41), over $m$ vanishes, leading to:

$$
b_{2, p m}^{0}=\frac{A_{0}}{2} \sum_{n} \frac{\sigma_{n}}{\epsilon_{p}}\left[-\eta \frac{\mathcal{K}_{m n p}}{\mathcal{N}_{m n p}^{2}} \zeta_{y, m n p} \zeta_{z, m n p}\right] a_{0, n p}^{0} .
$$


For all other surface current density expansion coefficients one finds similar results. In short, we can write the relation between $a_{0, n p}^{0}$ and any other nonzero expansion coefficient $b_{i, m n p}^{j}$ on face $S_{i}$ along its first $(j=0)$ or second axis $(j=1)$ as

$$
b_{i, m n p}^{j}=\Omega_{i, m n p}^{j}\left(\frac{A_{0}}{2} \frac{\sigma_{n}}{\epsilon_{p}}\right) a_{0, n p}^{0},
$$

for the same face $S_{0}$ and the opposite face $S_{1}$, where $\Omega_{i, m n p}^{j}$ evaluates to a single infinite sum (as for example shown in (41) and (45)) and to

$$
b_{i, m n p}^{j}=\sum_{n \text { or } p} \Upsilon_{i, m n p}^{j}\left(\frac{A_{0}}{2} \frac{\sigma_{n}}{\epsilon_{p}}\right) a_{0, n p}^{0},
$$

for the remaining faces where the summation runs over $n$ for $S_{2}$ and $S_{3}$ and over $p$ for $S_{4}$ and $S_{5}$. Note, however, that $\Upsilon_{i, m n p}^{j}$ is a known scalar (as e.g., for $S_{2}$ in (50)). Note as well that the bracketed term is independent of either $i$ or $j$ and only linked to the face and direction of $a_{0, n p}^{0}$.

At this point, we have fully described the relation between one set of electric field expansion coefficients on $S_{0}$ and all relevant current density coefficients on all faces. The same approach can now be repeated for all the other faces and directions to obtain a complete global relation between all expansion coefficients of both quantities. Clearly, the results are the same as described above apart from the required cyclic permutation of the axes and the employed indices. In summary, when we collect all coefficients into the vectors $\mathbf{a}_{c}$ and $\mathbf{b}_{c}$, we get

$$
\mathbf{b}_{c}=\overline{\bar{Y}}_{c} \mathbf{a}_{c}=\overline{\bar{X}} \overline{\bar{D}} \mathbf{a}_{c}
$$

with the expansion matrix $\overline{\bar{X}}$ being a sparse matrix whose nonzero entries are the - still to be evaluated $-\Omega_{i, m n p}^{j}$ and the already known scalar values $\Upsilon_{i, m n p}^{j}$, and where $\overline{\bar{D}}$ is a diagonal matrix with elements similar to the bracketed term in (51) and (52).

\section{B. Explicit analytic expressions}

Although all elements in $\overline{\bar{Y}}_{c}$ (or in particular in $\overline{\bar{X}}$ ) are theoretically fully defined, the infinite sum that still appears in $\Omega_{i, m n p}^{j}$ poses a computational challenge. However, we will now show that this sum possesses a closed analytical form, as such avoiding a cut-off of the sum and the corresponding loss in accuracy in the actual implementation, especially at high frequencies when accurate skin effect modeling is imperative.

As a first example, we examine the $\Omega_{i, m n p}^{j}$ values for (41). Adding up the contributions from both the TE and TM modes, we get

$$
\begin{aligned}
\Omega_{i, m n p}^{j} & =\frac{-4 \eta}{V} \sigma_{n}\left[\sum_{m=0}^{\infty} \frac{\epsilon_{m} \lambda_{y}^{2} \lambda_{z}^{2}}{\left(k^{2}-k_{m n p}^{2}\right)\left(k_{0}^{2}-k_{m n p}^{2}\right)\left(\lambda_{x}^{2}+\lambda_{y}^{2}\right)}\right. \\
& \left.+\epsilon_{p} \sum_{m=1}^{\infty} \frac{k_{m n p}^{2} \lambda_{x}^{2}}{\left(k^{2}-k_{m n p}^{2}\right)\left(k_{0}^{2}-k_{m n p}^{2}\right)\left(\lambda_{x}^{2}+\lambda_{y}^{2}\right)}\right] .
\end{aligned}
$$

By extracting the $m=0$ term of the first sum, recombining both fractions by the following substitutions:

$$
\begin{array}{r}
\alpha=\frac{l_{x}}{\pi} \alpha^{\prime}=\frac{l_{x}}{\pi} \sqrt{k^{2}-\lambda_{y}^{2}-\lambda_{z}^{2}} \\
\alpha_{0}=\frac{l_{x}}{\pi} \alpha_{0}^{\prime}=\frac{l_{x}}{\pi} \sqrt{k_{0}^{2}-\lambda_{y}^{2}-\lambda_{z}^{2}} \\
\theta=\frac{l_{x}}{\pi} \lambda_{y}=n l_{x} / l_{y} \quad \vartheta=\frac{l_{x}}{\pi} \lambda_{z}=p l_{x} / l_{z}
\end{array}
$$

we get the following expression for (54)

$$
\begin{aligned}
& \frac{-4 \eta}{V} \sigma_{n} \frac{l_{x}^{2}}{\pi^{2}}\left[\vartheta^{2}\left(\frac{1}{\alpha^{2} \alpha_{0}^{2}}+2 \sum_{m=1}^{\infty} \frac{1}{\left(m^{2}-\alpha^{2}\right)\left(m^{2}-\alpha_{0}^{2}\right)}\right)\right. \\
& \left.+\epsilon_{p} \sum_{m=1}^{\infty} \frac{m^{2}}{\left(m^{2}-\alpha^{2}\right)\left(m^{2}-\alpha_{0}^{2}\right)}\right] .
\end{aligned}
$$

The sums in this expression have closed analytical forms as outlined in Appendix A:

$$
\begin{aligned}
\Omega_{i, m n p}^{j} & =\frac{-4 \eta}{V} \sigma_{n} \frac{l_{x}^{2}}{\pi^{2}}\left[2 \vartheta^{2} \Omega_{0}\left(\alpha, \alpha_{0}\right)+\epsilon_{p} \Omega_{2}\left(\alpha, \alpha_{0}\right)\right] . \\
& =\frac{2 \sigma_{n} \epsilon_{p} l_{x}}{j \omega \mu_{0} V}\left[\frac{k^{2}-\lambda_{y}^{2}}{\alpha^{\prime}} \cot (\pi \alpha)-\frac{k_{0}^{2}-\lambda_{y}^{2}}{\alpha_{0}^{\prime}} \cot \left(\pi \alpha_{0}\right)\right]
\end{aligned}
$$

Second, the same procedure can be applied to the sum in (42). In this case, the TM contribution vanishes as $\zeta_{z}^{\mathrm{TM}} \equiv 0$, yielding

$$
\begin{aligned}
\Omega_{i, m n p}^{j} & =-\frac{4 \eta}{V} \sum_{m=0}^{\infty} \frac{\epsilon_{m} \lambda_{y} \lambda_{z}}{\left(k^{2}-k_{m n p}^{2}\right)\left(k_{0}^{2}-k_{m n p}^{2}\right)} \\
& =-\frac{4 \eta}{V} \frac{l_{x}^{2}}{\pi^{2}} \theta \vartheta\left[2 \Omega_{0}\left(\alpha, \alpha_{0}\right)\right] \\
& =\frac{4 l_{x}}{j \omega \mu_{0} V} \lambda_{y} \lambda_{z}\left[\frac{1}{\alpha^{\prime}} \cot (\pi \alpha)-\frac{1}{\alpha_{0}^{\prime}} \cot \left(\pi \alpha_{0}\right)\right]
\end{aligned}
$$

Third, as observed before (see (45) and (46)), very similar sums appear for parallel, non-coinciding planes. For example, the $\Omega_{i, m n p}^{j}$ value in (46) becomes:

$$
\begin{aligned}
\Omega_{i, m n p}^{j} & =\frac{4 \eta}{V} \sum_{m=0}^{\infty} \frac{\epsilon_{m}(-1)^{m} \lambda_{y} \lambda_{z}}{\left(k^{2}-k_{m n p}^{2}\right)\left(k_{0}^{2}-k_{m n p}^{2}\right)} \\
& =\frac{4 \eta}{V} \frac{l_{x}^{2}}{\pi^{2}} \theta \vartheta\left[2 \Psi_{0}\left(\alpha, \alpha_{0}\right)\right] \\
& =-\frac{4 l_{x}}{j \omega \mu_{0} V} \lambda_{y} \lambda_{z}\left[\frac{1}{\alpha^{\prime}} \csc (\pi \alpha)-\frac{1}{\alpha_{0}^{\prime}} \csc \left(\pi \alpha_{0}\right)\right]
\end{aligned}
$$

where we observe that the only difference with (61) is a minus sign and another type of auxiliary function, i.e., $\Psi_{n}(\cdot, \cdot)$ instead of $\Omega_{n}(\cdot, \cdot)$, to account for the $(-1)^{m}$ factor in the sum.

Above, we have demonstrated the existence of closed-form expressions for some specific examples. The same procedure is followed for all other expansion coefficients and, as expected from the symmetry of the problem, the resulting expressions are all of the same form provided that the correct cyclic permutation of the axes and indices is performed. At this point, 


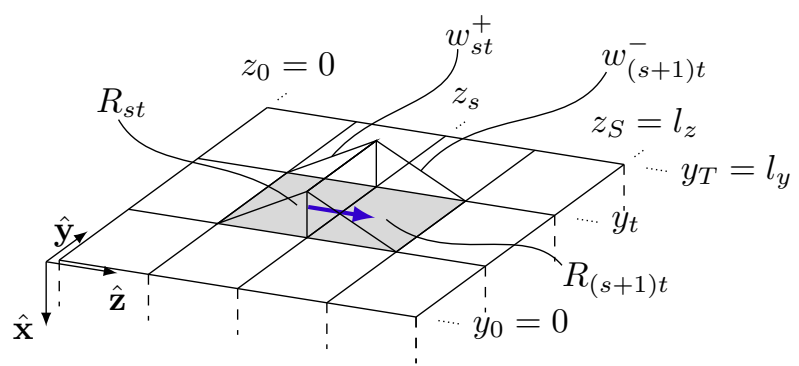

Fig. 2. Definition of a rooftop along $\hat{\mathbf{z}}$ on face $S_{0}$.

it is important to stress that $\overline{\bar{Y}}_{c}$ in (53) is free from infinite sums and, thus, efficiently and accurately maps the expansions coefficients $\mathbf{a}_{c}$ onto $\mathbf{b}_{c}$.

\section{DISCRETIZED $\mathcal{Y}$-OPERATOR FOR A CUBOID}

In Section III we derived an analytical form of the differential surface admittance operator for a cuboid based on entire domain basis functions. To solve the exterior problem, this operator is now integrated into a general boundary integral equation approach. Generally, these types of problems are solved employing local basis functions such as RWGs [51] or rooftop functions on a surface mesh. As we are dealing with cuboids to later on model interconnects, we opt for rooftop functions as they fit naturally on a rectangular surface grid and align with the trigonometric expansion of the relevant quantities. Restricting ourselves once more to $S_{0}$ and the $\hat{\mathbf{z}}$ component on this face, we expand $e_{z}=\mathbf{e} \cdot \hat{\mathbf{z}}$ as

$$
S_{0}: e_{z}=\sum_{s=1}^{S} \sum_{t=1}^{T} A_{0, s t}^{0} w_{s t}^{+}+A_{0,(s-1) t}^{0} w_{s t}^{-},
$$

with $S$ and $T$ the number of divisions along $\hat{\mathbf{z}}$ and $\hat{\mathbf{y}}$, respectively. The positive and negative half-rooftop functions $w_{s t}^{+}$and $w_{s t}^{-}$, as illustrated in Fig. 2, are defined as

$$
\begin{array}{ll}
w_{s t}^{+}=\frac{z-z_{s-1}}{\Delta z \Delta y}, & \{y, z\} \in R_{s t} \\
w_{s t}^{-}=\frac{z_{s}-z}{\Delta z \Delta y}, & \{y, z\} \in R_{s t},
\end{array}
$$

with $R_{s t}$ the rectangle that supports the half-rooftop function (see Fig. 2) and $\Delta z$ and $\Delta y$ its length along the $z$ - and $y$-axis, respectively. This expansion should equal the $z$-component of (36):

$$
S_{0}: e_{z}=\sum_{n^{\prime}, p^{\prime}} a_{0, n^{\prime} p^{\prime}}^{0} \sin \left(\lambda_{y}^{\prime} y\right) \cos \left(\lambda_{z}^{\prime} z\right)
$$

In order to connect expansions (65) and (68), we project them both onto $\sin \left(\lambda_{y} y\right) \cos \left(\lambda_{z} z\right) \hat{\mathbf{z}}$. For (68), the resulting integral resembles (37) and results in $a_{0, n p}^{0} A_{0} \sigma_{n} /\left(2 \epsilon_{p}\right)$. For (65), one gets,

$$
\sum_{s=1}^{S} \sum_{t=1}^{T} A_{0, s t}^{0} I_{s t}^{+}+A_{0,(s-1) t}^{0} I_{s t}^{-},
$$

with

$$
I_{s t}^{ \pm}=\int_{R_{s t}} w_{s t}^{ \pm} \sin \left(\lambda_{y} y\right) \cos \left(\lambda_{z} z\right) \mathrm{d} S=\phi_{y} \phi_{z}^{ \pm},
$$

and

$$
\begin{aligned}
\phi_{y} & =\frac{-1}{\Delta y \lambda_{y}}\left[\cos \left(\lambda_{y} y_{t}\right)-\cos \left(\lambda_{y} y_{t-1}\right)\right] \\
\phi_{z}^{+} & =\frac{\sin \left(\lambda_{z} z_{s}\right)}{\lambda_{z}}+\frac{\cos \left(\lambda_{z} z_{s}\right)-\cos \left(\lambda_{z} z_{s-1}\right)}{\lambda_{z}^{2} \Delta z} \\
\phi_{z}^{-} & =-\frac{\sin \left(\lambda_{z} z_{s-1}\right)}{\lambda_{z}}-\frac{\cos \left(\lambda_{z} z_{s}\right)-\cos \left(\lambda_{z} z_{s-1}\right)}{\lambda_{z}^{2} \Delta z} .
\end{aligned}
$$

When we generalize the results obtained above to all directions and faces and collect all rooftop basis function expansion coefficients (of the type $A_{0, s t}^{0}$ in (65)) into the vector $\mathbf{E}$, we get the following relation:

$$
\overline{\bar{M}} \mathbf{E}=\overline{\bar{D}} \mathbf{a}_{c},
$$

where the matrix $\overline{\bar{M}}$ links the rooftop and trigonometric functions via expression of the type (69) and the previously introduced diagonal scaling matrix $\overline{\bar{D}}$ (see (53)) is present on the right-hand side.

For the surface current density, we start with similar expansions:

$$
\begin{aligned}
S_{0}: j_{s, z} & =\sum_{s=1}^{S} \sum_{t=1}^{T} B_{0, s t}^{0} w_{s t}^{+}+B_{0,(s-1) t}^{0} w_{s t}^{-}, \\
S_{0}: j_{s, z} & =\sum_{n^{\prime \prime}, p^{\prime \prime}} b_{0, n^{\prime \prime} p^{\prime \prime}}^{0} \sin \left(\lambda_{y}^{\prime \prime} y\right) \cos \left(\lambda_{z}^{\prime \prime} z\right),
\end{aligned}
$$

which we now project onto rooftop testing functions $\mathbf{w}_{s^{\prime} t^{\prime}}=$ $\left[w_{s^{\prime} t^{\prime}}^{+}+w_{s^{\prime} t^{\prime}}^{-}\right] \hat{\mathbf{z}}$. For (76) this results in an integral of the same form as in (70) thus giving the following result for this procedure:

$$
\overline{\bar{M}}^{T} \mathbf{b}_{c} \text {. }
$$

Projecting (75) onto rooftops, results in a Gramian matrix $\overline{\bar{G}}$. Due to the local nature of the rooftops and the orthogonality of basis functions along different axes, only three elements per row/column are nonzero in $\overline{\bar{G}}$ : two entries for partially overlapping rooftops and one for the self-interaction. Therefore, only two distinct integrals are required:

$$
\begin{aligned}
\int_{R_{s t}} w_{s t}^{+} w_{s t}^{+} \mathrm{d} S & =\int_{R_{s t}} w_{s t}^{-} w_{s t}^{-} \mathrm{d} S=\frac{A_{R_{s t}}}{3(\Delta y)^{2}}, \\
\int_{R_{s t}} w_{s t}^{+} w_{s(t-1)}^{-} \mathrm{d} S & =\frac{A_{R_{s t}}}{6(\Delta y)^{2}},
\end{aligned}
$$

with $A_{R_{s t}}$ being the area of $R_{s t}$.

Consequently, the relation between both expansions of $\mathbf{j}_{s}$ is

$$
\overline{\bar{G}} \mathbf{I}=\overline{\bar{M}}^{T} \mathbf{b}_{c}
$$

where I collects all rooftop function expansion coefficients (of the type $B_{0, s t}^{0}$ in (75)) of $\mathbf{j}_{s}$. Combining (74) and (80) together with (53), finally yields

$$
\begin{aligned}
\mathbf{I} & =\overline{\bar{G}}^{-1} \overline{\bar{M}}^{T} \overline{\bar{Y}}_{c} \overline{\bar{D}}^{-1} \overline{\bar{M}} \mathbf{E} \\
& =\overline{\bar{G}}^{-1}\left(\overline{\bar{M}}^{T} \overline{\bar{X}} \overline{\bar{M}}\right) \mathbf{E} \\
& =\overline{\bar{G}}^{-1} \overline{\bar{Y}} \mathbf{E},
\end{aligned}
$$




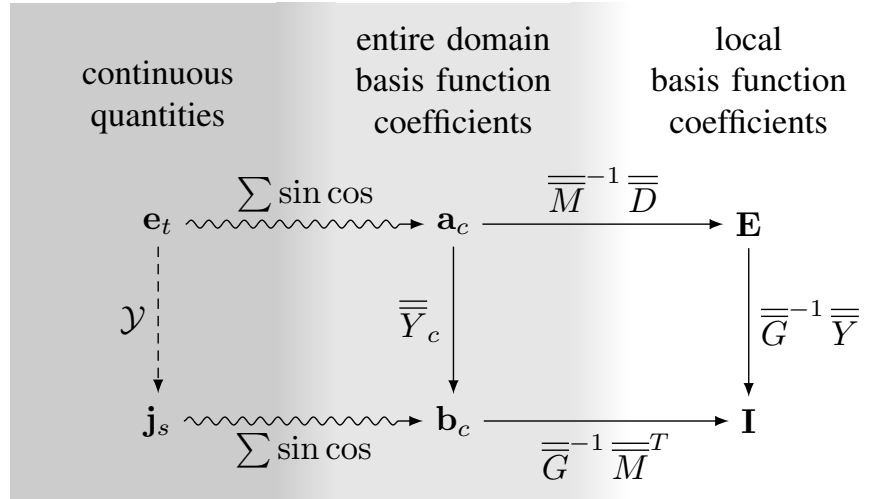

Fig. 3. Schematic overview of the discretization procedure.

with $\overline{\bar{Y}}$ now being the discrete version of (29). The entire procedure leading to (83) has been schematically summarized in Fig. 3. We conclude this section with some remarks about $\overline{\bar{Y}}$. All material properties and frequency dependencies are encapsulated in $\overline{\bar{X}}$. This implies that the calculation of the total matrix for various frequencies and/or materials only calls for separate instances of $\overline{\bar{X}}$ while $\overline{\bar{M}}$ can be reused. Similarly, all the mesh information is captured by $\overline{\bar{M}}$. The size of $\overline{\bar{X}}$ (and thus of $\overline{\bar{M}}$ ) depends on the number of entire domain basis functions that are taken into account. We will further denote this by the triplet $\{M, N, P\}$, indicating the maximum values taken by $m, n$ and $p$.

\section{Determination of THE IMPEDANCE RESPONSE}

In this section, we utilize the differential surface admittance operator in a framework to calculate the impedance of 3$\mathrm{D}$ interconnect structures. For this purpose, we extend the approach of [43], where the resistance and inductance were calculated using a circuit interpretation of the electric field integral equation (EFIE), to also including capacitive couplings. Circuit interpretations of integral equations techniques were first presented in [7], leading to the PEEC method. In the end, this leads to a matrix formulation similar to the augmented EFIE formulation [47], albeit including losses. The starting point is the EFIE for the equivalent problem depicted in Fig. 1(b):

$$
\mathbf{e}=-j \omega \mathbf{a}-\nabla \phi,
$$

with $\mathbf{a}$ and $\phi$ being the magnetic vector potential and electric scalar potential, respectively. Note that we have assumed that no incident source fields are present as we are dealing with interconnects. Discretizing (84) involves choosing basis and test functions. We opt for the earlier introduced rooftop functions for both, in other words, we will apply Galerkin weighting.

For the left-hand side, this procedure leads to $\overline{\bar{G}} \mathbf{E}$ analogous to the projection of the rooftop expansion of $\mathbf{j}_{s}$ in Section IV. Note that for multiple objects, $\bar{G}$ is block diagonal with the Gramiam matrix of each separate object on its diagonal since each basis function is restricted to its respective object.
The vector potential is given by

$$
\mathbf{a}(\mathbf{r})=\mu_{0} \int_{\cup \mathcal{S}_{i}} G\left(\mathbf{r}, \mathbf{r}^{\prime}\right) \mathbf{j}_{s}\left(\mathbf{r}^{\prime}\right) \mathrm{d} S^{\prime},
$$

where the integral is taken over the boundaries of all objects and $G\left(\mathbf{r}, \mathbf{r}^{\prime}\right)$ is the Green's function of the background medium into which the objects are embedded. For the examples presented further in this paper, we limit ourselves to free space with its corresponding static Green's function:

$$
G\left(\mathbf{r}, \mathbf{r}^{\prime}\right)=\frac{1}{4 \pi\left|\mathbf{r}-\mathbf{r}^{\prime}\right|}
$$

By substituting the rooftop expansion of $\mathbf{j}_{s}$ and Galerkin testing, the matrix equivalent of (85) becomes:

$$
\begin{aligned}
& \int_{\mathcal{S}_{f}} \mathbf{a}(\mathbf{r}) \cdot \mathbf{w}_{f}(\mathbf{r}) \mathrm{d} S \\
& =\mu_{0} \sum_{g} I_{g} \int_{S_{f}} \int_{S_{g}} G\left(\mathbf{r}, \mathbf{r}^{\prime}\right) \mathbf{w}_{f}(\mathbf{r}) \cdot \mathbf{w}_{g}\left(\mathbf{r}^{\prime}\right) \mathrm{d} S \mathrm{~d} S^{\prime} \\
& =\sum_{g}(\overline{\bar{L}})_{f g} I_{g},
\end{aligned}
$$

with $S_{i}$ the support of the basis/testing function $\mathbf{w}_{i}$. Repeating the above for all $\mathbf{w}_{f}$, the discretized version of the first term in the right-hand side of (84) becomes $-j \omega \overline{\bar{L}} \mathbf{I}$ with $\overline{\bar{L}}$ the inductance matrix and $\mathbf{I}$ the vector collecting all coefficients $I_{g}$. Matrix $\overline{\bar{L}}$ is dense since every basis function is interacting with all others through the Green's function. Projecting the last term in (84) on the rooftops and employing partial integration and the divergence theorem leads to

$$
\int_{\mathcal{S}_{f}} \nabla \phi \cdot \mathbf{w}_{f} \mathrm{~d} S=\oint_{c_{f}} \underbrace{\phi \hat{\mathbf{n}} \cdot \mathbf{w}_{f}}_{=0} \mathrm{~d} c-\int_{R_{f}^{+}} \frac{\phi}{A_{f}^{+}} \mathrm{d} S+\int_{R_{f}^{-}} \frac{\phi}{A_{f}^{-}} \mathrm{d} S,
$$

where $A_{f}^{ \pm}$is the area of $R_{f}^{ \pm}$, the two rectangles that make up the support of $\mathbf{w}_{f}$. By introducing (83), (84) is discretized as

$$
\left(\overline{\bar{G}} \overline{\bar{Y}}^{-1} \overline{\bar{G}}+j \omega \overline{\bar{L}}\right) \mathbf{I}-\overline{\bar{T}} \mathbf{V}=0,
$$

where $\overline{\bar{Y}}$ is the differential surface admittance operator for multiple objects. This generalized matrix is, just as $\bar{G}$, block diagonal with the discretized $\mathcal{Y}$-operator of each object on its diagonal. The vector $\mathbf{V}$ and incidence matrix $\overline{\bar{T}}$, which maps the patches and edges of the mesh, are defined as

$$
\begin{gathered}
(\mathbf{V})_{g}=\int_{R_{g}} \frac{\phi}{A_{g}} \mathrm{~d} S, \\
(\overline{\bar{T}})_{f g}= \begin{cases}1, & \text { if } R_{g} \text { is } R_{f}^{+} \text {of } \mathbf{w}_{f} \\
-1, & \text { if } R_{g} \text { is } R_{f}^{-} \text {of } \mathbf{w}_{f} \\
0, & \text { otherwise, }\end{cases}
\end{gathered}
$$

with $R_{g}$ being a rectangle of the surface mesh with area $A_{g}$.

To account for the capacitive effects, note that the scalar potential is related to the divergence of the surface current density through

$$
\phi(\mathbf{r})=\frac{-1}{j \omega \epsilon_{0}} \int_{\cup \mathcal{S}_{i}} G\left(\mathbf{r}, \mathbf{r}^{\prime}\right) \nabla \cdot \mathbf{j}_{s}\left(\mathbf{r}^{\prime}\right) \mathrm{d} S^{\prime} .
$$




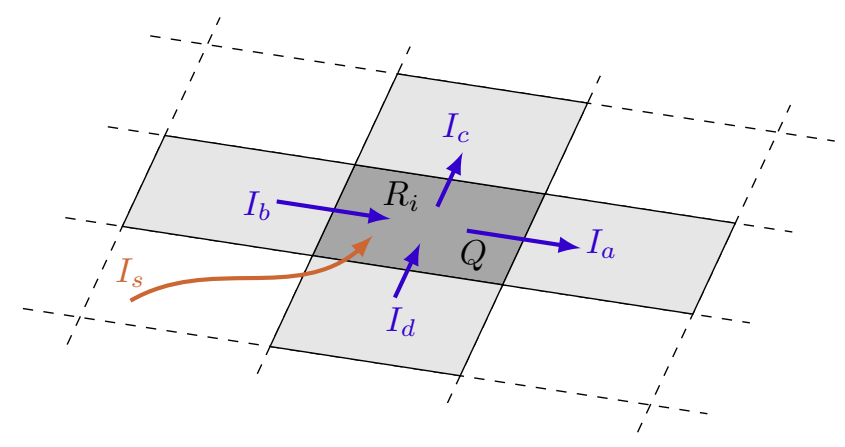

Fig. 4. Charge conservation on a single rectangle $R_{i}$. The blue arrows represent the various rooftop functions that contribute to the charge $Q$ on the dark gray rectangle while the orange arrow is a (possible) external current source.

Inserting the piecewise constant divergence of the basis functions and averaging over the rectangles of the mesh, leads to the following matrix relation

$$
\mathbf{V}=\overline{\bar{K}} \mathbf{Q},
$$

with the column vector $\mathbf{Q}$ collecting the charges on each rectangle and the elements of $\overline{\bar{K}}$ given by

$$
(\overline{\bar{K}})_{f g}=\frac{1}{\epsilon_{0}} \int_{R_{f}} \int_{R_{g}} \frac{G\left(\mathbf{r}, \mathbf{r}^{\prime}\right)}{A_{f} A_{g}} \mathrm{~d} S \mathrm{~d} S^{\prime} .
$$

To eliminate $\mathbf{Q}$, we discretize the conservation of charge law on every rectangle. As shown for an arbitrary rectangle $R_{i}$ in Fig. 4, various currents (can) contribute to a single rectangle: rooftops defined on adjacent patches and possibly external current sources. The charge conservation equation $\nabla \cdot \mathbf{j}_{s}+j \omega \rho_{s}=0$ on $R_{i}$ then becomes

$$
I_{a}-I_{b}+I_{c}-I_{d}+j \omega Q=I_{s} .
$$

Generalizing this relation to all mesh elements, leads to the matrix equation

$$
\overline{\bar{T}}^{T} \mathbf{I}+j \omega \mathbf{Q}=\mathbf{S},
$$

where the vector $\mathbf{S}$ contains the external current sources.

Combining (90) together with (94) and (97), leads to the following set of matrix equations

$$
\left[\begin{array}{cc}
\overline{\bar{G}} \overline{\bar{Y}}^{-1} \overline{\bar{G}}+j \omega \overline{\bar{L}} & -\overline{\bar{T}} \\
\overline{\bar{T}}^{T} & j \omega \overline{\bar{K}}
\end{array}\right]\left[\begin{array}{l}
\mathbf{I} \\
\mathbf{V}
\end{array}\right]=\left[\begin{array}{l}
\mathbf{0} \\
\mathbf{S}
\end{array}\right] .
$$

This equation can subsequently be solved by a direct or iterative solver. At this point, however, (98) represents a set of separate objects residing in a background medium without any interconnection. In order to represent realistic structures, the various cuboids should be combined. We achieve this by introducing an infinitesimally small PEC wire connection between adjacent rectangles of two cuboids which equates the voltages of the adjacent rectangles and introduces an extra, yet unknown current flowing integrally from one rectangle to the other (for more detail, see Appendix B). This is implemented in the matrix system (98) by introducing an extra row with only two nonzero entries 1 and -1 to equate the voltages and an extra column with only two nonzero entries 1 and -1

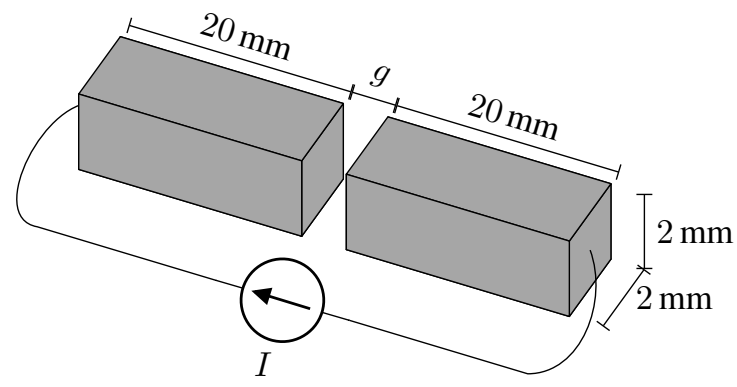

Fig. 5. Two copper blocks $(2 \times 2 \times 20 \mathrm{~mm})$ separated by a variable distance $g$.

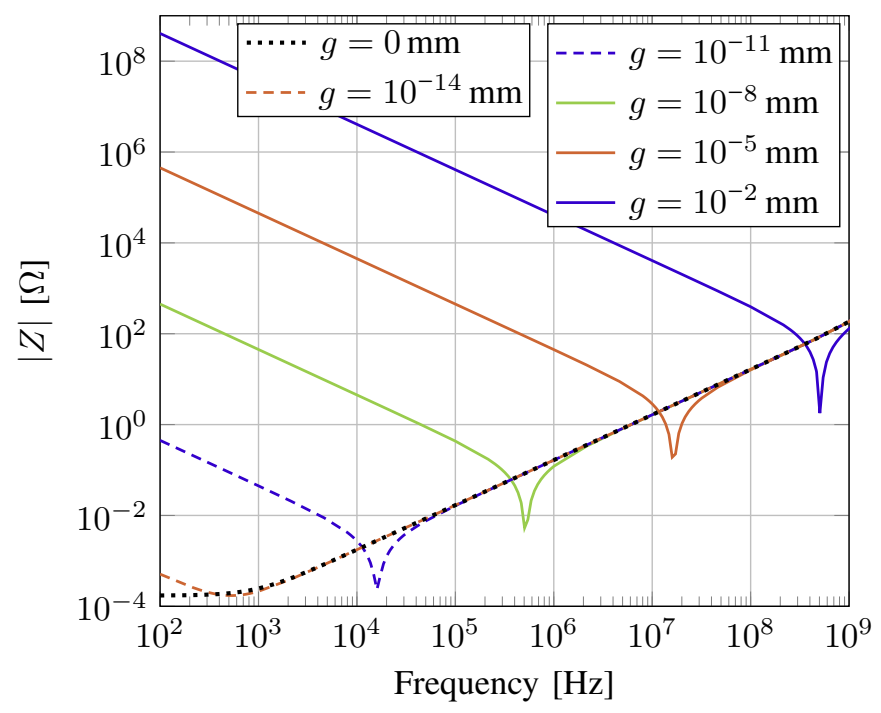

Fig. 6. Magnitude of the total impedance of the two copper blocks shown in Fig. 5 as a function of frequency for various separation distances $g$.

to add the additional current to the two charge conservation equations of the involved rectangles.

\section{NUMERICAL RESULTS}

\section{A. Validation examples}

The first example constitutes a simple structure consisting of two copper $\left(\sigma=5.8 \cdot 10^{7} \mathrm{~S} / \mathrm{m}\right)$ blocks with dimensions $2 \mathrm{~mm} \times 2 \mathrm{~mm} \times 20 \mathrm{~mm}$ as shown in Fig. 5. This example is not only used to validate the technique presented in this paper, but it is also employed to compare the newly proposed method to calculate $\bar{Y}$ with the technique presented in [43] in terms of accuracy, computation time and memory requirement. The total number of edges (and thus number of rooftops) is set to 572 in order to eliminate any coarse mesh effects in the analysis of the $\mathcal{Y}$-operator's accuracy.

Focusing on the structure first, we calculate its impedance by measuring the voltage over the terminals where the unit current source connects to the copper blocks. The magnitude of this impedance is shown in Fig. 6 for various values of the gap $g$. The black dotted line, i.e., for $g=0 \mathrm{~mm}$, corresponds to one single copper block of length $40 \mathrm{~mm}$ and is included as a reference result for the limit $g \rightarrow 0$. 


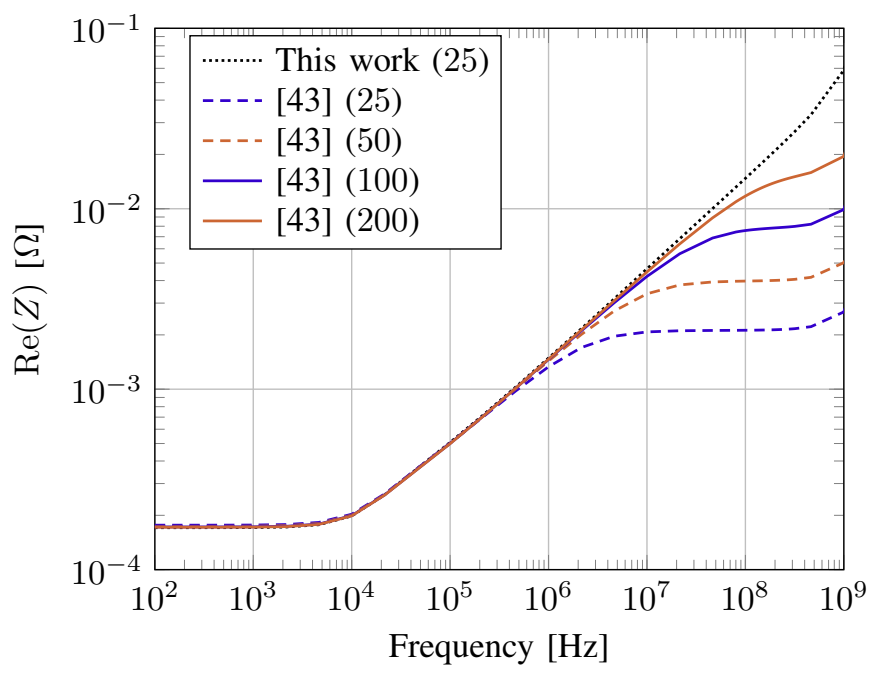

Fig. 7. Real part of the impedance for two copper blocks (see Fig. 5) as a function of frequency with $g=1 \mu \mathrm{m}$ for the approach presented in this work and in [43]. The number of entire domain basis functions used along $x, y$ and $z$-axis are $\{M, M, 25\}$ respectively (with both blocks aligned along the $z$-axis) and the employed value of $M$ is denoted between brackets.

For all nonzero values of $g$, a series resonance occurs due to the interplay of the inductance, which is the same for all configurations, and the capacitance of the gap, which decreases for increasing distances as such pushing the resonance frequency to higher values. Once beyond its self-resonance, the inductive behavior dominates and the impedance response coincides with the reference results regardless of the separation distance.

In order to assess the accuracy of the technique, we turn our attention to the real part of the impedance for a fixed separation distance $g$ of $1 \mu \mathrm{m}$. In Fig. 7, we clearly see that for low frequencies the method (black dotted curve) correctly predicts the Pouillet resistance value of $1.7 \cdot 10^{-4} \Omega$ and that it exhibits the characteristic $\sqrt{f}$-behavior as the skin effect develops. For this example the maximum value $M$ of the indices governing the cross-section, i.e., $m$ and $n$, has been set to 25 , with the same value along the longitudinal $z$-axis. These values were set heuristically as of yet no general criterion for these numbers has been found or developed. When we compare this to an earlier 3-D differential surface admittance operator for cuboids [43] with the same restrictions on the indices, we see that while the low frequency results coincide nicely, the resistance curve for the earlier version of the operator levels out around $10 \mathrm{MHz}$. This nonphysical result is caused by cutting off the infinite summation for numerical evaluation, which is clearly detrimental for accurate results, especially for a strongly developed skin effect. This leveling out would be alleviated by driving up the number of eigenmodes $M$ to higher values, but as shown in Fig. 7, even a considerable amount of eigenmodes does not suffice to yield adequate results over the complete frequency range. Moreover, it comes at a high computation cost. In the novel technique proposed here, this shortcoming is solved by exploiting the closed form of infinite sums as demonstrated in Section III-B.

Table I contains a more detailed comparison of the compu-
TABLE I

COMPUTATION TIME AND MEMORY USAGE PER FREQUENCY POINT FOR THE CONFIGURATION OF FIG. 7.

\begin{tabular}{llll}
\hline \hline Metric & Eq. (83) (25) & [43] (25) & {$[43](200)$} \\
\hline Number of edges & 572 & 572 & 572 \\
$\overline{\bar{Y}}$ calculation [s] & 0.86 & 0.88 & 30.91 \\
Matrix solution [s] & 0.5 & 0.51 & 0.5 \\
$\overline{\bar{Y}}$ calculation [MiB] & 5.6 & 5.1 & 5.5 \\
\hline
\end{tabular}

tation cost by listing the computation time per frequency point and memory consumption for the provided example. A set-up time of $170 \mathrm{~s}$ for the computation of the $\overline{\bar{L}}$ and $\overline{\bar{K}}$ matrices is not included as these results can be reused for all frequency points. From the table we clearly see that the computation time and memory requirements for both methods are of the same order for the same number of eigenmodes. However, when comparing to the result from [43] with an increased number of eigenmodes to increase accuracy at higher frequencies, the newly presented method evidently outperforms the other technique. The memory consumption stays roughly the same but the computation time increases by a factor 35 for an increase of $M$ from 25 to 200 .

At this point, we would like to clarify some points with regard to the mesh size required when employing the differential surface admittance operator. The typical rule of thumb of ten to twenty elements per wavelength of the background medium to achieve electrically small mesh elements, as is the case with any typical BIE method, applies. This is, of course, supplemented with the need to sufficiently approximate the (fine) geometrical details, often the limiting factor in interconnects and/or (on-chip) packaging. Importantly, the differential surface admittance operator negates the need for the mesh elements to be electrically small in the (conductive) medium with a substantially smaller wavelength, as the skin effect is fully encapsulated in the $\overline{\bar{Y}}$-matrix without invoking the Green's function inside the medium. As such, the presented method inherits the computation complexity properties of traditional BIE-MoM formalisms for scattering at perfect electrical conductors or of BIE-MoM solvers for interconnects where the conductivity is simply tackled via, e.g., a Leontovich surface impedance boundary condition. In other terms, the MoM-system of the presented method scales in exactly the same fashion. This also implies that the application of acceleration algorithms, e.g., the Fast Multipole Method (FMM), will yield the typical speed-ups as found in [52]. So, the advocated BIE-MoM technique accurately takes care of the skin effect, without compromising on efficiency, as the scaling properties of traditional BIE-MoM approaches are retained.

For the second example, consider the copper loop depicted in Fig. 8. It measures $1988 \mu \mathrm{m} \times 126 \mu \mathrm{m}$ with a square crosssection of $4 \mu \mathrm{m} \times 4 \mu \mathrm{m}$ [33]. The admittance of the structure is measured over the $0.1 \mu \mathrm{m}$ gap in the middle of the shorter arm. The real and imaginary part of the admittance are compared with the FastHenry [53] and GIBC [54] result and shown in Fig. 9. The number of edge elements (560) results in a mesh coarseness similar to the one reported in [33]. We observe 


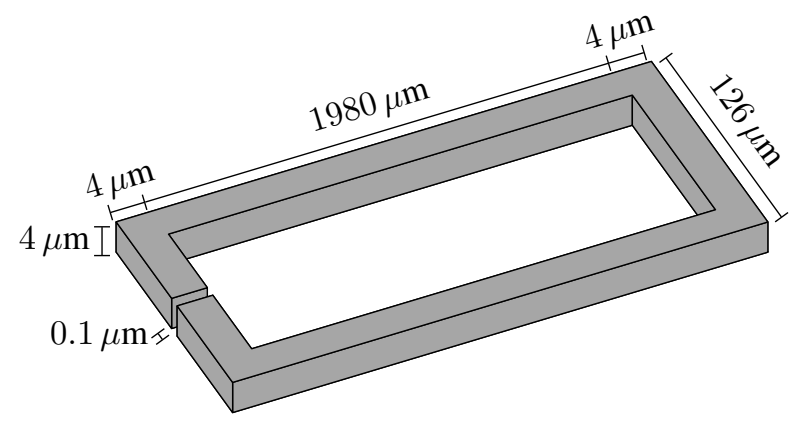

Fig. 8. Rectangular copper loop $(1988 \mu \mathrm{m} \times 126 \mu \mathrm{m})$ with a cross-section of $4 \mu \mathrm{m} \times 4 \mu \mathrm{m}[33]$.

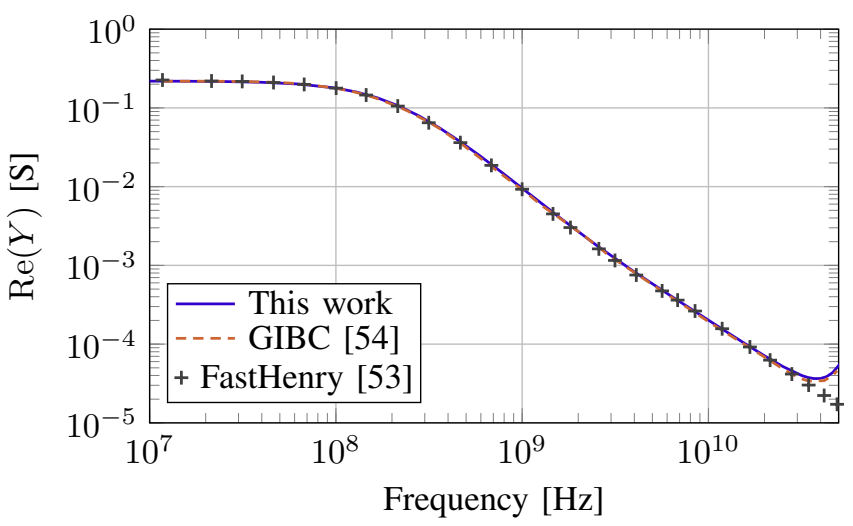

(a) Real part of the admittance expressed in S.

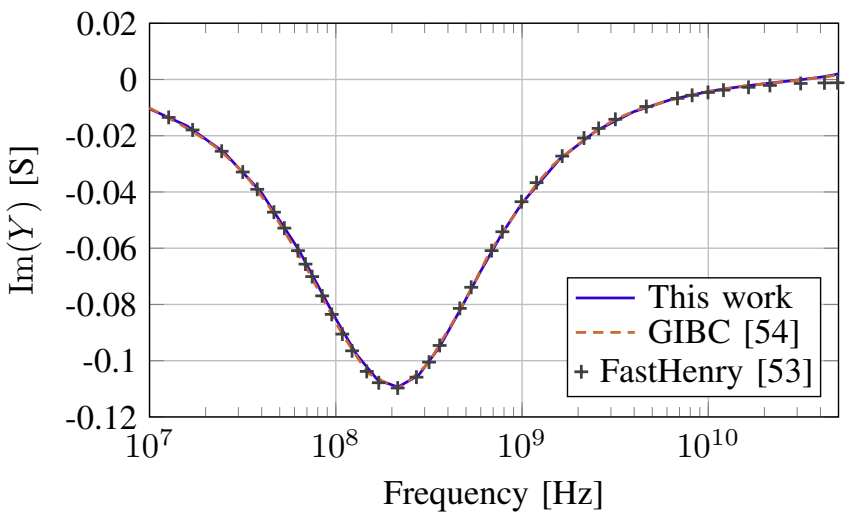

(b) Imaginary part of the admittance expressed in S.

Fig. 9. Admittance as a function of frequency for the geometry shown in Fig. 8, compared to the GIBC method [54] and FastHenry [53].

an excellent agreement between this work and the GIBC over the entire frequency range. The results obtained through FastHenry start to deviate from the other two at the higher end of the frequency range as capacitive interactions are not taken into account in this method. Details on the computation time and memory usage can be found in Table II.

\section{B. Application examples}

1) Square coil array: Consider an array of square, copper coils, arranged in a $3 \times 3$ grid with a mutual spacing of $30 \mu \mathrm{m}$ as described and simulated with VoxHenry in [55] (see Fig. 10). Each coil, having a square cross-section with side
TABLE II

COMPUTATION TIME AND MEMORY USAGE PER FREQUENCY POINT FOR THE CONFIGUR ATIONS IN Fig. 8, Fig. 10, Fig. 12 AND Fig. 14

\begin{tabular}{lllll}
\hline \hline Metric & Fig. 8 & Fig. 10 & Fig. 12 & Fig. 14 \\
\hline Number of edges & 560 & 3600 & 1360 & 3216 \\
Memory for $\overline{\bar{Y}}[\mathrm{MiB}]$ & 2.32 & 24.2 & 5.52 & 7.64 \\
$\overline{\bar{Y}}$ calculation [s] & 6.2 & 7.79 & 10.24 & 11.42 \\
Matrix solution [s] & 0.47 & 103.57 & 8.48 & 46.73 \\
\hline HFSS computation time [s] & - & 47.2 & - & 7.05 \\
ADS computation time [s] & - & - & 1.2 & - \\
\hline
\end{tabular}

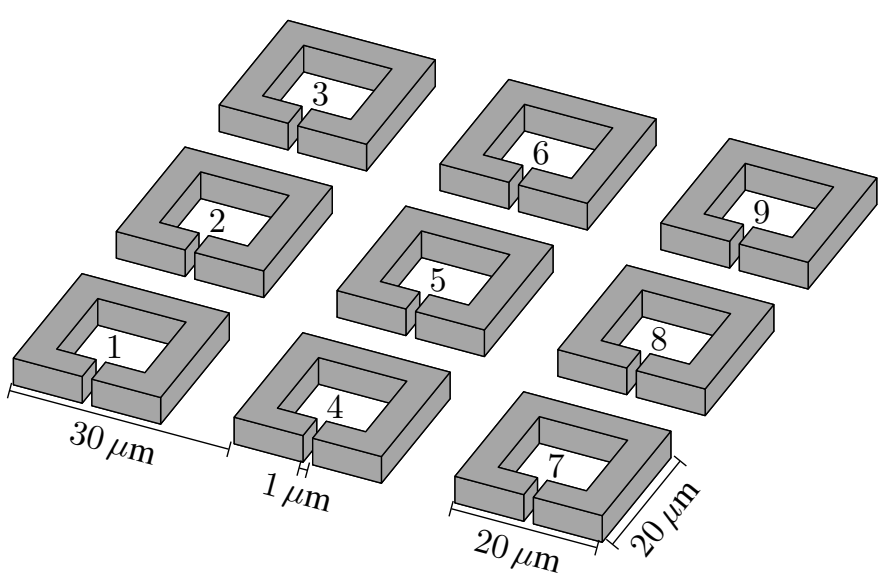

Fig. 10. $3 \times 3$ array of square, copper loops $(20 \mu \mathrm{m} \times 20 \mu \mathrm{m})$ with a square cross-section of $4 \mu \mathrm{m} \times 4 \mu \mathrm{m}$ spaced $30 \mu \mathrm{m}$ apart. A port is defined over a $1 \mu \mathrm{m}$ gap on the same side for every loop [55].

$4 \mu \mathrm{m}$, measures $20 \mu \mathrm{m} \times 20 \mu \mathrm{m}$. In the middle of one side of all coils, a port is defined over a $1 \mu \mathrm{m}$ gap. The array will be studied for two different cases, viz., with and without ground plane. The ground plane is an infinite PEC plane positioned $2 \mu \mathrm{m}$ below the loops. Both situations are compared to a corresponding ANSYS HFSS simulation [56]. Some details on the computation time and memory usage for the first case are shown in Table II.

In Fig. 11 a few selected items from the structure's Z-parameters are shown as a function of frequency. Fig. 11(a) shows the real part of $Z_{55}$, i.e., the resistance of the central loop. The proposed method, VoxHenry (see [55]) and HFSS all predict the same response although a small deviation is visible at higher frequencies between all three. This can be attributed to the inherent difference between volumetric, volume integral equation (VIE) and BIE methods. Nevertheless, it is apparent from the set of results that the ground plane has a negligible influence on the resistance.

Turning to Fig. 11(b), the imaginary part of the same Z-matrix element over $\omega$ is shown in $\mathrm{pH}$. The curves for the structure in free space both coincide very well with the VoxHenry result. The case with ground plane results in an offset between both curves. This deviation is caused by the different implementation of the infinite ground plane. In our BIE approach, this feature is taken into account through the Green's function while in the FEM based HFSS solver this is 


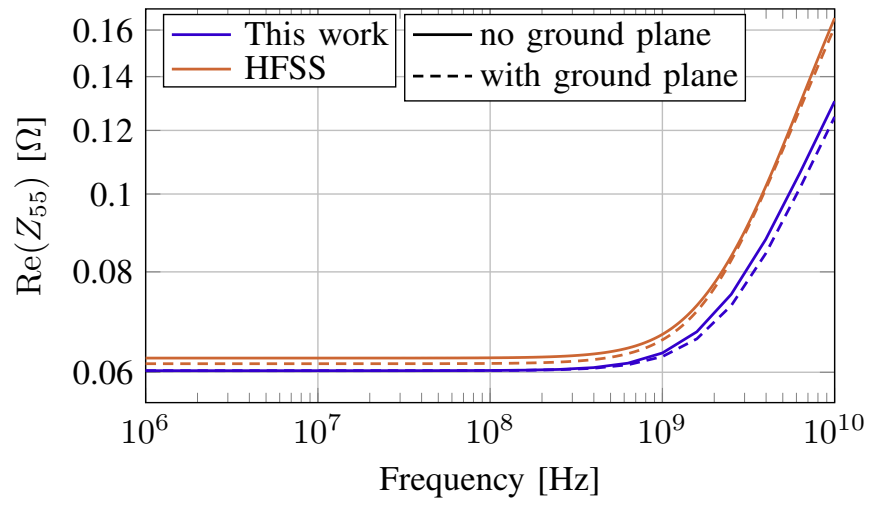

(a) Real part of $Z_{55}$ expressed in $\Omega$.

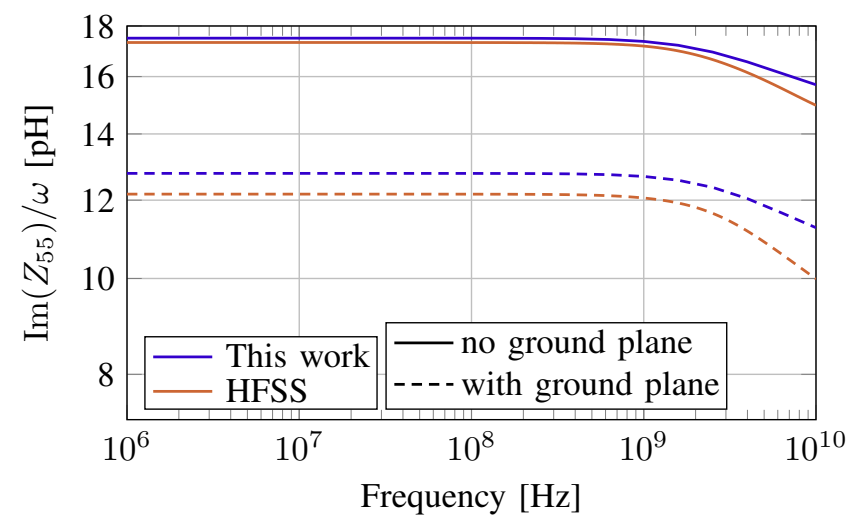

(b) Imaginary part of $Z_{55}$ over $\omega$ expressed in $\mathrm{pH}$.

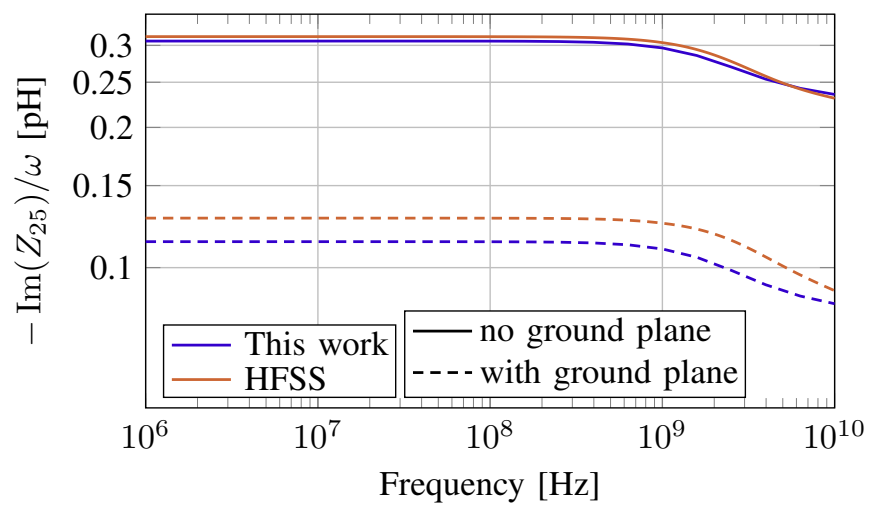

(c) The negative imaginary part of $Z_{25}$ over $\omega$ expressed in $\mathrm{pH}$.

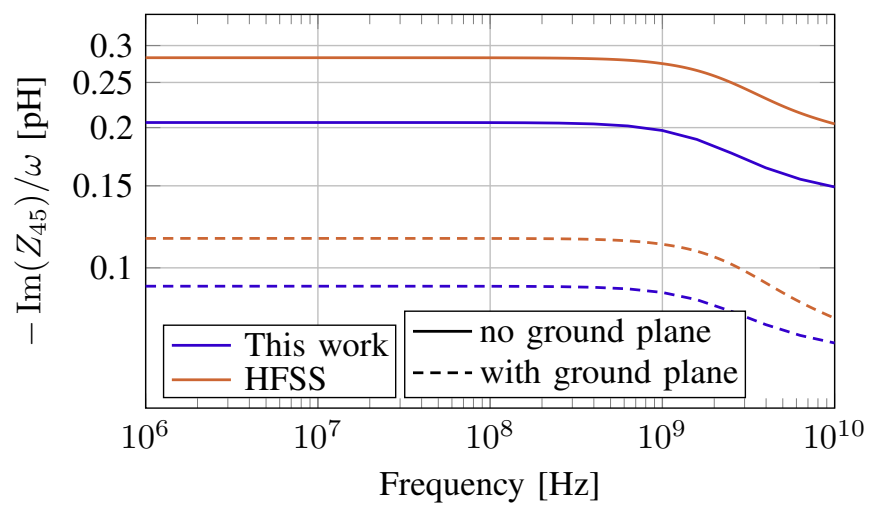

(d) The negative imaginary part of $Z_{45}$ over $\omega$ expressed in $\mathrm{pH}$.

Fig. 11. Selected impedance matrix elements as a function of frequency for the geometry shown in Fig. 10 with (solid) and without (dashed) ground plane.

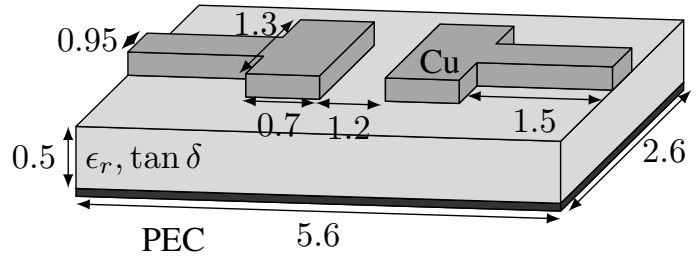

Fig. 12. Copper (thickness of $35 \mu \mathrm{m}$ ) footprint for an SMD resistor on an FR4 substrate $\left(\epsilon_{r}=4.2, \tan \delta=0.02\right)$. All dimensions are given in $\mathrm{mm}$.

achieved through the appropriate boundary conditions on the bounding box of the simulation domain. In [55] the shift in inductance due to the ground plane is not observed but can be attributed to the fact that their ground plane is finite and lossy.

Moving on to the off-diagonal elements, we take a closer look at the coupling between two sets of adjacent loops, i.e., loop two and loop five in Fig. 11(c) and loop four and loop five in Fig. 11(d), respectively. For the first pair, the correspondence between both solvers is similar to Fig. 11(b) while for the second pair there is a larger discrepancy. Comparing the two pairs, both our method and HFSS predict a smaller coupling for the second pair but using the differential surface admittance operator this drop is bigger, resulting in the observed difference in the response. A possible explanation for this disparity is the difference in port definition between the compared methods. Still, comparing to the VoxHenry results, we see that our response curves again coincide nicely for the free space case.

2) Surface-mount device footprint on a PCB substrate: The second application example constitutes a 0805 surfacemount device (SMD) footprint on a PEC-plane backed FR4 substrate with $\epsilon_{r}=4.2$ and a loss tangent of 0.02 . The traces are made out of copper with a thickness of $35 \mu \mathrm{m}$. The structure, depicted with all relevant dimensions in Fig. 12, is characterized with the presented technique and with Keysight ADS-Momentum, a 3-D planar BIE simulator [57], in the presence of a $10 \Omega$ resistor between the landing pads. In ADS- Momentum, the substrate is taken into account through the layered media Green's function, while in our work, the substrate is in fact modeled as a finite dielectric cuboid, characterized by means of the differential surface admittance operator, just as the conductors are. The resulting S-parameters are shown in Fig. 13 and calculated up to $13 \mathrm{GHz}$, the frequency above which ADS-Momentum warns that the port size grows too large (electrically) and the S-parameters can no longer be computed reliably. The results from both solvers agree very well over the entire frequency range except for the higher end $\left|S_{11}\right|$. This is due to the conductor modeling approach in the commercial solver, which uses a modified two-sheet current model that relies on heuristic broadband surface impedance expressions. Such models typically give good asymptotic results but are not particularly accurate at frequencies for which the skin effect is developing, which explains why the deviation in Fig. 13(b) occurs once the magnitude of $S_{11}$ starts increasing under the influence of the skin effect. 


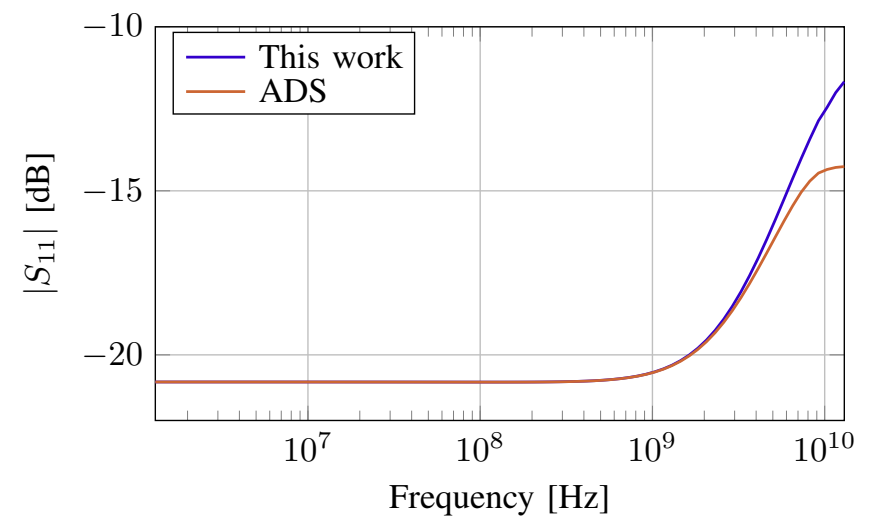

(a) $\left|S_{11}\right|$.

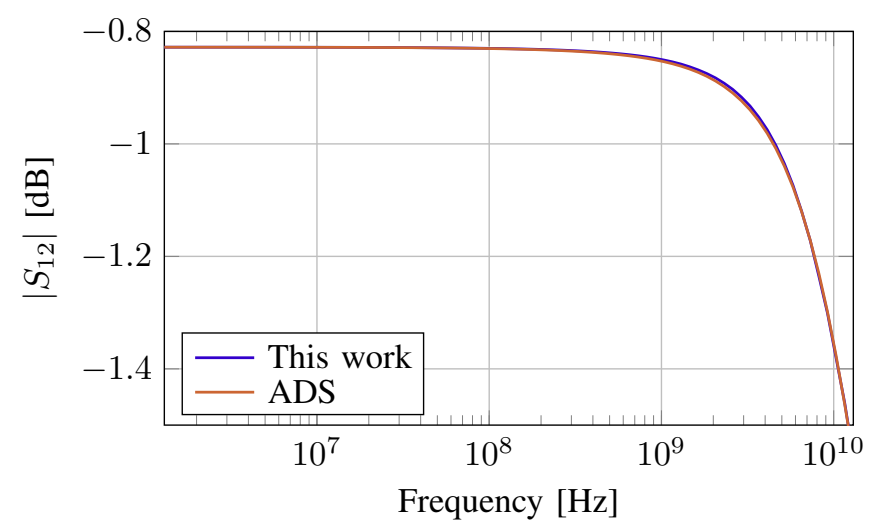

(b) $\left|S_{12}\right|$.

Fig. 13. S-parameters of the footprint shown in Fig. 12 with a resistor of $10 \Omega$ placed between both landing pads.

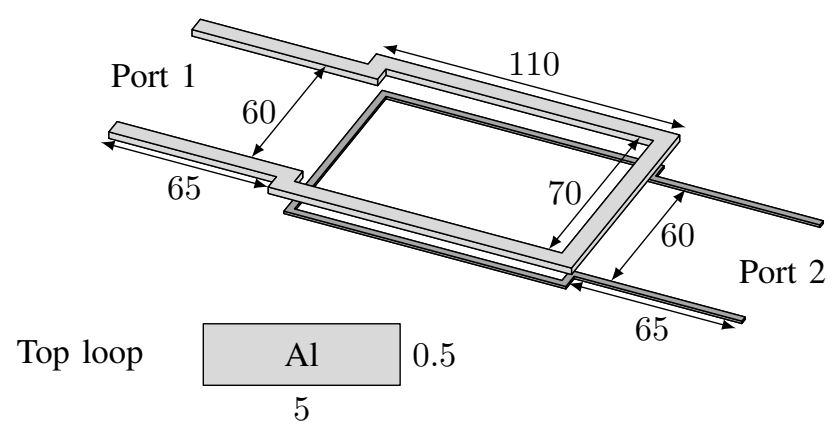

Bottom loop $\frac{\mathrm{Cu}}{2} 0.173$

Fig. 14. Two coupled loops spaced $6.508 \mu \mathrm{m}$ apart. The top aluminum loop $\left(\sigma=3.77 \cdot 10^{7} \mathrm{~S} / \mathrm{m}\right)$ has a cross-section of $5 \mu \mathrm{m} \times 0.5 \mu \mathrm{m}$ while the bottom copper loop measures $2 \mu \mathrm{m} \times 0.173 \mu \mathrm{m}$. All dimensions are given in $\mu \mathrm{m}$.

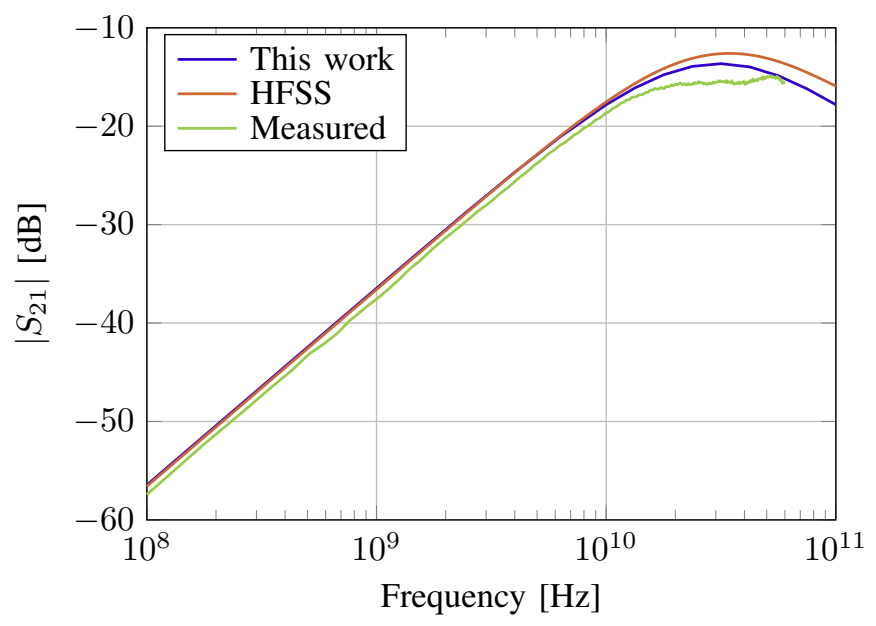

(a) Absolute value of $S_{21}$.

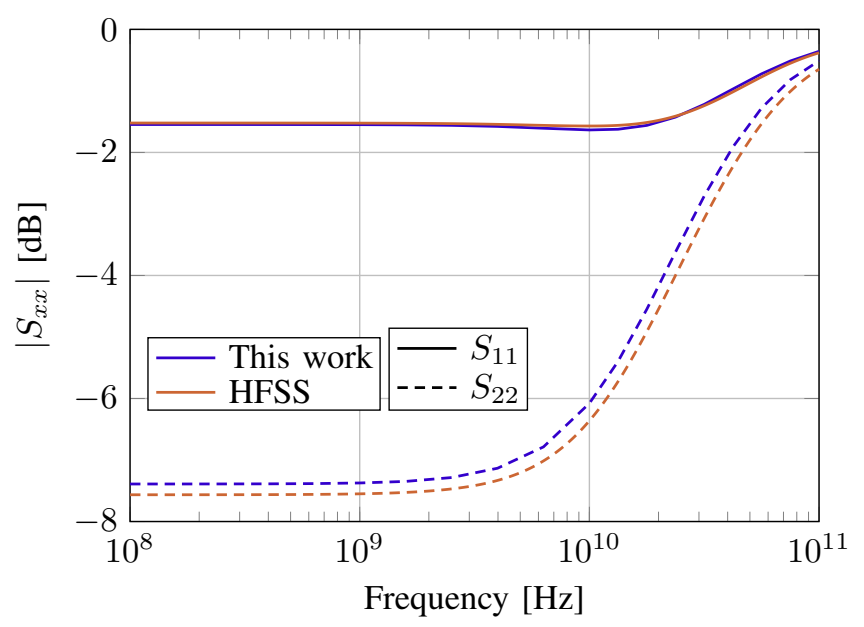

(b) Absolute value of $S_{11}$ and $S_{22}$.

Fig. 15. Selected scattering matrix elements as a function of frequency for the geometry shown in Fig. 14.

3) Two coupled on-chip inductors: As a final application example, a test chip was manufactured with an aluminum $\left(\sigma=3.77 \cdot 10^{7} \mathrm{~S} / \mathrm{m}\right)$ loop $6.508 \mu \mathrm{m}$ above a smaller copper loop. Both elements have the same inner dimensions for the loop, i.e., $100 \mu \mathrm{m} \times 70 \mu \mathrm{m}$, but have different cross-sections, as shown in Fig. 14 together with all other relevant dimensions, which are all given in micrometers. The loops are placed in an on-chip multi-layered stack-up that is not included here as the nonmagnetic materials have negligible influence on the mainly inductive coupling phenomena in this setup. The scattering parameters of this structure are simulated by means of the proposed method and HFSS (computation details can be found in Table II). The coupling is also compared to measurement data and shown in Fig. 15. The measured diagonal elements of the S-matrix are not included in the analysis as the complex feed structure and measurement setup did not allow for stable de-embedding over the entire frequency range.

Starting with Fig. 15(a), we observe excellent agreement between the proposed method, HFSS and the measured $\left|S_{21}\right|$. The measurement shows a slightly lower coupling over the entire frequency band due to additional losses in the mea- 
surement. At the end of the frequency range the three curves diverge more due to high frequency noise in the measurement data and the earlier observed difference between volumetric and surface methods. Advancing to Fig. 15(b), we show the absolute value of $S_{11}$ and $S_{22}$ for the coupled loops. Once again, we obtain very good agreement between our novel method and HFSS. A summary of the computation time and memory usage are reported in Table II. As was the case with the previous example in Section VI-B1, these figures show that the main cost per frequency point is given by the direct solution of the system matrix. For larger geometries, iterative matrix algorithms are required to avoid this step becoming a major bottleneck. As the EFIE is prone to conditioning problems, certainly at low frequencies, techniques to counteract this have to be employed. The augmented EFIE formulation provides various options to improve conditioning such as enforcing charge neutrality, scaling the various blocks and/or applying a preconditioner [47], [48]. Preliminary tests have shown that these methods remain effective when modifying the formulation to include losses as presented in Section V.

\section{CONCLUSION}

A boundary integral equation framework to accurately model good conductors is presented. By means of a new formulation of the differential surface admittance operator for cuboids, the behavior of lossy materials is taken into account more rigorously and efficiently than before. The inclusion of the operator into the augmented electric field integral equation leads to a full and stable characterization of interconnects over a broad frequency range. The method is thoroughly validated and compared to earlier work, commercial software and measurement data, demonstrating both its accuracy and appositeness.

\section{APPENDIX A}

\section{AUXILIARY FUNCTIONS}

\section{A. Closed analytical expressions for fundamental sums}

The various series in Section III-B can all be decomposed into the fundamental sums featured here. Their analytical expressions are calculated utilizing a contour integration method as outlined in the appendix of [58]:

$$
\begin{aligned}
\phi(x) & =\sum_{k=1}^{\infty} \frac{1}{\left(k^{2}-x^{2}\right)}=\frac{1}{2 x^{2}}[1-\pi x \cot (\pi x)] \\
\chi(x) & =\sum_{k=1}^{\infty} \frac{1}{\left(k^{2}-x^{2}\right)^{2}} \\
& =\frac{1}{4 x^{4}}\left[-2+\pi x \cot (\pi x)+(\pi x)^{2} \csc ^{2}(\pi x)\right] \\
\rho(x) & =\sum_{k=1}^{\infty} \frac{(-1)^{k}}{\left(k^{2}-x^{2}\right)}=\frac{1}{2 x^{2}}[1-\pi x \csc (\pi x)] \\
\varsigma(x) & =\sum_{k=1}^{\infty} \frac{(-1)^{k}}{\left(k^{2}-x^{2}\right)^{2}} \\
& =\frac{1}{4 x^{4}}[-2+\pi x \csc (\pi x)(1+\pi x \cot (\pi x))]
\end{aligned}
$$




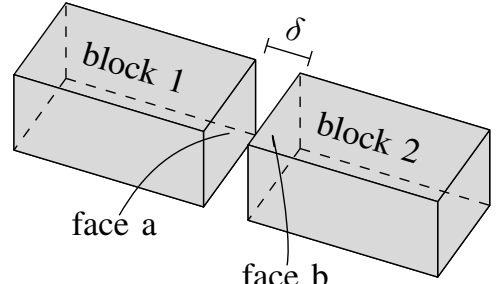

Fig. 16. Two cuboids separated by a small gap $\delta$.

$$
\begin{aligned}
& \Omega_{0}(a, a)=\frac{1}{2 a^{4}}+\chi(a)=\frac{\pi}{4 a^{3}}\left[\cot (\pi a)+\pi a \csc ^{2}(\pi a)\right] \\
& \Omega_{2}(a, a)=a^{2} \chi(a)+\phi(a)=\frac{\pi}{4 a}\left[-\cot (\pi a)+\pi a \csc ^{2}(\pi a)\right] \\
& \Psi_{0}(a, a)=\frac{1}{2 a^{4}}+\varsigma(a)=\frac{\pi}{4 a^{3}} \csc (\pi a)[\pi a \cot (\pi a)+1] \\
& \Psi_{2}(a, a)=a^{2} \varsigma(a)+\rho(a)=\frac{\pi}{4 a} \csc (\pi a)[\pi a \cot (\pi a)-1]
\end{aligned}
$$

\section{APPENDIX B}

\section{CUBOID INTERCONNECTION}

For completeness, below, we further clarify the theoretical reasoning which shows that connecting several cuboids with (infinitesimally short) wires leads to the correct results, as also discussed in [43].

The 3-D differential surface admittance operator enforces a relation between the differential surface current density (introduced after application of the equivalence principle) and the electric field on the boundary. This relation is theoretically exact [5] and fully captures the effects of the replaced medium. The subdivision of the structure does initially introduce approximations but if the junctions are modeled properly and connected with a number of short wires, the entire structure will be fully and correctly taken into account.

We have demonstrated before, i.e., in [43] (in particular the examples in Figs. 4 and 5 in that paper) the rigor of this method, showing that the interplay of the 3-D differential surface admittance operator and this way to interconnect the various cuboids enables accurate characterization for true 3-D structures.

The way to understand why the wire interconnections rigorously model the junction, leading to the presented good results, is the following (for two interconnected blocks). Suppose the blocks are initially separated by a small gap $\delta$ between face a of block 1 and face b of block 2 as depicted in Fig. 16. First, the technique models the two separate blocks exactly by, and this is important, taking all interactions between the blocks into account. In this way, we have modeled two pieces of good conductors in which a total current is injected in block 1 and extracted from block 2 . The gap essentially constitutes a capacitance between the blocks. To connect the blocks $(\delta=0)$ the potentials $V$ of corresponding mesh points in faces a and $\mathrm{b}$ are taken to be identical by short-circuiting them. This is established on the equivalent network level. As a consequence, (a) the gap capacitance no longer comes into play (no potential difference) and (b) by leaving the value of the current flowing through the short circuits free, i.e., their values will follow from the circuit solution, the law of conservation of charge is also meticulously enforced. Hence, a correct modeling of the combination of the two blocks is obtained. The above reasoning of course applies to a chain of blocks.

\section{REFERENCES}

[1] P. Arlett, A. Bahrani, and O. Zienkiewicz, "Application of finite elements to the solution of Helmholtz's equation," Proc. Inst. Electr. Eng., vol. 115, no. 12, p. 1762, Dec. 1968.

[2] G. Costache, "Finite element method applied to skin-effect problems in strip transmission lines," IEEE Trans. Microw. Theory Techn., vol. 35, no. 11, pp. 1009-1013, Nov. 1987.

[3] M. Tsuk and J. Kong, "A hybrid method for the calculation of the resistance and inductance of transmission lines with arbitrary cross sections," IEEE Trans. Microw. Theory Techn., vol. 39, no. 8, pp. 13381347, Aug. 1991.

[4] R.-B. Wu and J.-C. Yang, "Boundary integral equation formulation of skin effect problems in multiconductor transmission lines," IEEE Trans. Magn., vol. 25, no. 4, pp. 3013-3015, Jul. 1989.

[5] D. De Zutter and L. Knockaert, "Skin effect modeling based on a differential surface admittance operator," IEEE Trans. Microw. Theory Techn., vol. 53, no. 8, pp. 2526-2538, Aug. 2005.

[6] T. Demeester and D. De Zutter, "Quasi-TM transmission line parameters of coupled lossy lines based on the Dirichlet to Neumann boundary operator," IEEE Trans. Microw. Theory Techn., vol. 56, no. 7, pp. 16491660, Jul. 2008.

[7] A. Ruehli, "Equivalent circuit models for three-dimensional multiconductor systems," IEEE Trans. Microw. Theory Techn., vol. 22, no. 3, pp. 216-221, Mar. 1974

[8] K. Coperich, A. Ruehli, and A. Cangellaris, "Enhanced skin effect for partial-element equivalent-circuit (PEEC) models," IEEE Trans. Microw. Theory Techn., vol. 48, no. 9, pp. 1435-1442, Sep. 2000.

[9] U. R. Patel and P. Triverio, "Skin effect modeling in conductors of arbitrary shape through a surface admittance operator and the contour integral method," IEEE Trans. Microw. Theory Techn., vol. 64, no. 9, pp. 2708-2717, Sep. 2016.

[10] Y. Zhao, F. Ling, and J. Mao, "Novel surface impedance modeling for broadband parameter extraction of 3-D interconnects," IEEE Microw. Wireless Compon. Lett., vol. 27, no. 1, pp. 7-9, Jan. 2017.

[11] J. Jin, Theory and Computation of Electromagnetic Fields. Wiley, 2011.

[12] J. Volakis, A. Chatterjee, and L. Kempel, Finite Element Method Electromagnetics: Antennas, Microwave Circuits, and Scattering Applications, ser. IEEE Press Series on Electromagnetic Wave Theory. Wiley, 1998.

[13] M. Al-Qedra, J. Aronsson, and V. Okhmatovski, "A novel skin-effect based surface impedance formulation for broadband modeling of 3-D interconnects with electric field integral equation," IEEE Trans. Microw. Theory Techn., vol. 58, no. 12, pp. 3872-3881, Dec. 2010.

[14] S. Omar and D. Jiao, "A new volume integral formulation for broadband 3-D circuit extraction in inhomogeneous materials with and without external electromagnetic fields," IEEE Trans. Microw. Theory Techn., vol. 61, no. 12, pp. 4302-4312, Dec. 2013.

[15] F. Sheikh Hosseini Lori, A. Menshov, R. Gholami, J. B. Mojolagbe, and V. Okhmatovski, "Novel single-source surface integral equation for scattering problems by 3-D dielectric objects," IEEE Trans. Antennas Propag., vol. 66, no. 2, pp. 797-807, Feb. 2018.

[16] J. Nitsch, F. Gronwald, and G. Wollenberg, Radiating Nonuniform Transmissionline Systems and the Partial Element Equivalent Circuit Method. Chichester, UK: John Wiley \& Sons, Ltd, Oct. 2009.

[17] A. Poggio and E. Miller, Integral Equation Solutions of Threedimensional Scattering Problems. Pergamon, 1973, ch. 4, pp. 159-264.

[18] S. Chakraborty and V. Jandhyala, "Evaluation of Green's function integrals in conducting media," IEEE Trans. Antennas Propag., vol. 52, no. 12 , pp. 3357-3363, Dec. 2004.

[19] J. Peeters, I. Bogaert, and D. De Zutter, "Calculation of MoM interaction integrals in highly conductive media," IEEE Trans. Antennas Propag., vol. 60, no. 2, pp. 930-940, Feb. 2012.

[20] M. Gossye, M. Huynen, D. Vande Ginste, D. De Zutter, and H. Rogier, "A Calderón preconditioner for high dielectric contrast media," IEEE Trans. Antennas Propag., vol. 66, no. 2, pp. 808-818, Feb. 2018. 
[21] P. Ylä-Oijala, J. Markkanen, S. Järvenpää, and S. P. Kiminki, "Surface and volume integral equation methods for time-harmonic solutions of Maxwell's equations," Prog. Electromagn. Res., vol. 149, no. Aug., pp. 15-44, 2014.

[22] P. Ylä-Oijala, S. P. Kiminki, K. Cools, F. P. Andriulli, and S. Järvenpää, "Mixed discretization schemes for electromagnetic surface integral equations," Int. J. Numer. Model. Electron. Networks, Devices Fields, vol. 25, no. 5-6, pp. 525-540, Sep. 2012.

[23] K. A. Michalski and D. Zheng, "Electromagnetic scattering and radiation by surfaces of arbitrary shape in layered media. I. Theory," IEEE Trans. Antennas Propag., vol. 38, no. 3, pp. 335-344, 1990.

[24] C.-T. Tai, Dyadic Green functions in electromagnetic theory. IEEE Press, 1994.

[25] F. Ling, D. Jiao, and J.-M. Jin, "Efficient electromagnetic modeling of microstrip structures in multilayer media," IEEE Trans. Microw. Theory Techn., vol. 47, no. 9, pp. 1810-1818, 1999.

[26] W.-h. Tang and S. D. Gedney, "An efficient application of the discrete complex image method for quasi-3-D microwave circuits in layered media," IEEE Trans. Microw. Theory Techn., vol. 55, no. 8, pp. 17231729, Aug. 2007.

[27] K. Yang and A. E. Yilmaz, "A three-dimensional adaptive integral method for scattering from structures embedded in layered media," IEEE Trans. Geosci. Remote Sens., vol. 50, no. 4, pp. 1130-1139, Apr. 2012.

[28] Z. Zhu, B. Song, and J. White, "Algorithms in FastImp: a fast and wideband impedance extraction program for complicated 3-D geometries," IEEE Trans. Comput.-Aided Design Integr. Circuits Syst., vol. 24, no. 7, pp. 981-998, Jul. 2005.

[29] T. Senior and J. Volakis, Approximate Boundary Conditions in Electromagnetics, ser. Electromagnetics and Radar Series. Institution of Electrical Engineers, 1995.

[30] Dau-Sing Wang, "Limits and validity of the impedance boundary condition on penetrable surfaces," IEEE Trans. Antennas Propag., vol. 35, no. 4, pp. 453-457, Apr. 1987.

[31] Y. Zhao and J. Mao, "Equivalent surface impedance-based mixed potential integral equation accelerated by optimized $\mathcal{H}$-matrix for 3 -D interconnects," IEEE Trans. Microw. Theory Techn., vol. 66, no. 1, pp. 22-34, Jan. 2018

[32] Z.-G. Qian, W. C. Chew, and R. Suaya, "Generalized impedance boundary condition for conductor modeling in surface integral equation," IEEE Trans. Microw. Theory Techn., vol. 55, no. 11, pp. 2354-2364, Nov. 2007.

[33] Z.-G. Qian, M.-S. Tong, and W. C. Chew, "Conductive medium modeling with an augmented GIBC formulation," Prog. Electromagn. Res., vol. 99, pp. 261-272, 2009.

[34] T. Xia, H. Gan, M. Wei, W. C. Chew, H. Braunisch, Z. Qian, K. Aygun, and A. Aydiner, "An integral equation modeling of lossy conductors with the enhanced augmented electric field integral equation," IEEE Trans. Antennas Propag., vol. 65, no. 8, pp. 4181-4190, Aug. 2017.

[35] T. Demeester and D. De Zutter, "Construction of the Dirichlet to Neumann boundary operator for triangles and applications in the analysis of polygonal conductors," IEEE Trans. Microw. Theory Techn., vol. 58, no. 1, pp. 116-127, Jan. 2010.

[36] U. R. Patel, B. Gustavsen, and P. Triverio, "Proximity-aware calculation of cable series impedance for systems of solid and hollow conductors,' IEEE Trans. Power Del., vol. 29, no. 5, pp. 2101-2109, Oct. 2014.

[37] H. Ameri and R. Faraji-Dana, "Differential global surface impedance (DGSI): a rigorous model for analyzing periodic structures," J. Opt. Soc. Am. B, vol. 34, no. 5, p. 930, May 2017.

[38] U. R. Patel, P. Triverio, and S. V. Hum, "A novel single-source surface integral method to compute scattering from dielectric objects," IEEE Antennas Wireless Propag. Lett., vol. 16, pp. 1715-1718, 2017.

[39] U. R. Patel, S. V. Hum, and P. Triverio, "A magneto-quasi-static surface formulation to calculate the impedance of 3D interconnects with arbitrary cross-section," in 2017 IEEE 21st Workshop on Signal and Power Integrity (SPI), May 2017, pp. 1-4.

[40] U. R. Patel, S. Sharma, S. Yang, S. V. Hum, and P. Triverio, "Full-wave electromagnetic characterization of 3D interconnects using a surface integral formulation," in 2017 IEEE 26th Conf. Electr. Perform. Electron. Packag. Syst., Oct. 2017, pp. 1-3.

[41] M. Huynen, M. Gossye, D. De Zutter, and D. Vande Ginste, "A 3-D differential surface admittance operator for lossy dipole antenna analysis," IEEE Antennas Wireless Propag. Lett., vol. 16, pp. 1052 $1055,2017$.

[42] M. Huynen, D. De Zutter, and D. Vande Ginste, "Boundary integral equation study of the influence of finite conductivity on antenna radiation using a 3-D differential surface admittance operator," in 2017 Inter- national Applied Computational Electromagnetics Society Symposium Italy (ACES), Mar. 2017

[43] - "Rigorous full-wave resistance and inductance computation of 3-D interconnects," IEEE Microw. Wireless Compon. Lett., vol. 28, no. 6, pp. 455-457, Jun. 2018.

[44] _ "A fully 3-D BIE evaluation of the resistance and inductance of on-board and on-chip interconnects," in 2018 IEEE 22nd Work. Signal Power Integr., no. 1, May 2018, pp. 1-4.

[45] — "Broadband full-wave BIE impedance characterization of 3-D interconnects," in 2018 IEEE 27th Conference on Electrical Performance of Electronic Packaging and Systems (EPEPS), Oct. 2018, pp. 241-243.

[46] M. Huynen, K. Y. Kapusuz, D. De Zutter, and D. Vande Ginste, "An enhanced differential surface admittance operator for the signal integrity modeling of interconnects," in 2019 Int. Conf. Electromagn. Adv. Appl. IEEE, Sep. 2019, pp. 60-65.

[47] Z.-G. Qian and W. C. Chew, "An augmented electric field integral equation for high-speed interconnect analysis," Microw. Opt. Technol. Lett., vol. 50, no. 10, pp. 2658-2662, Oct. 2008.

[48] — "Fast full-wave surface integral equation solver for multiscale structure modeling," IEEE Trans. Antennas Propag., vol. 57, no. 11, pp. 3594-3601, Nov. 2009.

[49] J. Van Bladel, Electromagnetic Fields. John Wiley \& Sons, 2007.

[50] K. Kurokawa, "The expansions of electromagnetic fields in cavities," IRE Trans. Microw. Theory Tech., vol. 6, no. 2, pp. 178-187, Apr. 1958.

[51] S. Rao, D. Wilton, and A. Glisson, "Electromagnetic scattering by surfaces of arbitrary shape," IEEE Trans. Antennas Propag., vol. 30, no. 3, pp. 409-418, May 1982.

[52] W. C. Chew, J.-M. Jin, E. Michielssen, and J. Song, Fast and Efficient Algorithms in Computational Electromagnetics. Artech House, 2001.

[53] M. Kamon, J. K. White, and M. J. Tsuk, "FASTHENRY: A multipoleaccelerated 3-D inductance extraction program," IEEE Trans. Microw. Theory Techn., vol. 42, no. 9, pp. 1750-1758, 1994.

[54] W. C. Chew, M. S. Tong, and B. Hu, "Integral equation methods for electromagnetic and elastic waves," Synth. Lect. Comput. Electromagn., vol. 3, no. 1, pp. 1-241, Jan. 2008

[55] A. C. Yucel, I. P. Georgakis, A. G. Polimeridis, H. Bağcı, and J. K. White, "VoxHenry: FFT-accelerated inductance extraction for voxelized geometries," IEEE Trans. Microw. Theory Techn., vol. 66, no. 4, pp. 1723-1735, Apr. 2018

[56] ANSYS HFSS. [Online]. Available: https://www.ansys.com/

[57] Keysight Momentum 3D Planar EM Simulator. [Online]. Available: https://www.keysight.com/en/pc-1887116/momentum-3d-planarem-simulator

[58] R. Collin, Field Theory of Guided Waves. IEEE Press, 1991.

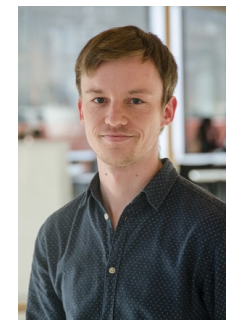

Martijn Huynen (S'18 - M'19) received the M.Sc. degree and Ph.D. degree in electrical engineering from Ghent University, Gent, Belgium in 2014 and 2019, respectively.

$\mathrm{He}$ is currently working as a Post-Doctoral researcher at the Electromagnetics Group/IDLab in the Department of Information Technology of Ghent University/imec. His research focuses on computational electromagnetics, more specifically boundary integral equations, with applications in interconnect modeling, electromagnetic compatibility anal-

ysis and antenna design.

Dr. Huynen received the Best Student Paper Award at the 2017 Applied Computational Electromagnetics Society (ACES) Symposium. 


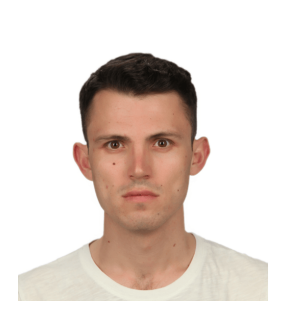

Kamil Yavuz Kapusuz (S'17) was born in 1988. He received the M.Sc. degree in electrical engineering from Atilim University, Ankara, Turkey in 2013. $\mathrm{He}$ is currently pursuing the Ph.D. degree in electrical engineering with the Electromagnetics Group, Department of Information Technology, Ghent, Belgium.

From 2014 to 2016, he was with Remote Sensing Technologies, Ankara, Turkey. He developed antenna arrays at millimeter-wave frequencies for radar applications. He also developed scalable phased array antennas for SATCOM-on-the-move concepts. His research interests include design and development of passive RF components, multifunction and reconfigurable antennas, active antennas, and phased arrays.

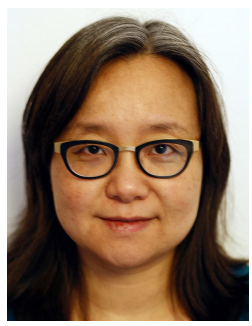

Xiao Sun received a Ph.D. degree in Electrical Engineering in 2001 from Université Grenoble Alpes, Grenoble, France. Since then, she has been a senior scientist at imec, Belgium. Her research interests include RF design, modeling and characterization for 3D interconnects and their impact on 3D ICs. She is also actively involved in the heterogeneous integration and packaging for RF and 5G applications. She has (co)authored over 70 peer-reviewed journals and conference papers and one book chapter. She was the recipient of the prestigious outstanding session paper award from ECTC 2015.

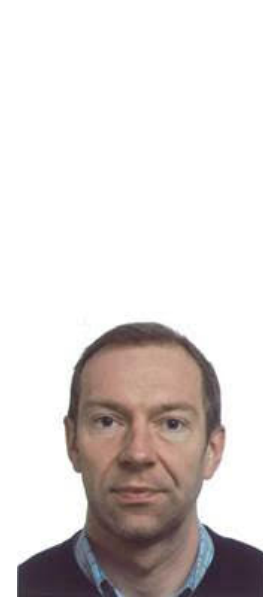

Geert Van der Plas obtained Ph.D. degree from the Katholieke Universiteit Leuven, Belgium, in 2001. $\mathrm{He}$ joined imec, Belgium, in 2003.

$\mathrm{He}$ has been working on energy efficient data converter, power/signal integrity and 3D integration technologies.

Currently he is program manager in the $3 \mathrm{D}$ program addressing system scaling using advanced 3D (TSV) and packaging (FO-WLP) technology for high performance, mobile and IoT applications. His interests are in characterization, modeling, system exploration and design enablement of 3D integration technologies.

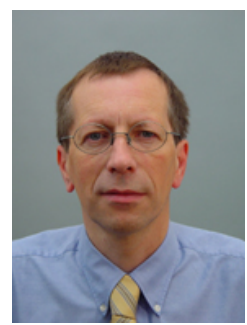

Daniël De Zutter was born in 1953. He received his M. Sc. Degree in electrical engineering from the University of Gent in 1976. In 1981 he obtained a Ph. D. degree and in 1984 he completed a thesis leading to a degree equivalent to the French Aggrégation or the German Habilitation (both at the University of Gent).

$\mathrm{He}$ was a full professor of electromagnetics. His research focusses on all aspects of circuit and electromagnetic modelling of high-speed and highfrequency interconnections and packaging, on Electromagnetic Compatibility (EMC) and numerical solutions of Maxwell's equations. As author or co-author he has contributed to more than 220 international journal papers (cited in the Web of Science) and 250 papers in conference proceedings. In 2000 he was elected to the grade of Fellow of the IEEE. He was an Associate Editor for the IEEE Microwave Theory and Techniques Transactions. Between 2004 and 2008 he served as the Dean of the Faculty of Engineering of Ghent University and he was the head of the Department of Information Technology between 2010 and 2016.

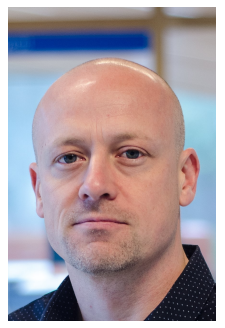

Dries Vande Ginste (SM'12) received the M.S. degree and the Ph.D. degree in electrical engineering from Ghent University, Gent, Belgium, in 2000 and 2005, respectively. He is currently an Associate Professor at the Department of Information Technology, Ghent University and a Guest Professor at imec. In June and July 2004, he was a Visiting Scientist at the Department of Electrical and Computer Engineering, University of Illinois at UrbanaChampaign (UIUC), IL, USA. From September to November 2011, he was a Visiting Professor at the EMC Group, Dipartimento di Elettronica, Politecnico di Torino, Italy. $\mathrm{He}$ has authored or co-authored over 200 papers in international journals and in conference proceedings. His research interests include computational electromagnetics, electromagnetic compatibility, signal and power integrity, and antenna design. Dr. Vande Ginste was awarded the International Union of Radio Science (URSI) Young Scientist Award at the 2011 URSI General Assembly and Scientific Symposium, the Best Poster Paper Award at the 2012 IEEE Electrical Design of Advanced Packaging and Systems Symposium (EDAPS), the Best Paper Award at the 2013 IEEE Workshop on Signal and Power Integrity (SPI), the Best Paper Award at the 2013 IEEE International Conference on Electrical Performance of Electronic Packaging and Systems (EPEPS) and the Best Paper Award at the 2016 IEEE Electrical Design of Advanced Packaging and Systems Symposium (EDAPS). He served as the chair of the 2014 IEEE Workshop on Signal and Power Integrity. He is a Senior Member of the IEEE.

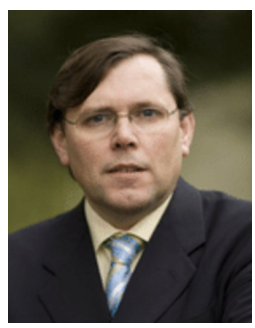

Eric Beyne (M'83 - SM'17) received the master's degree in electrical engineering and the Ph.D. degree in applied sciences from KU Leuven, Leuven, Belgium, in 1983 and 1990, respectively.

Since 1986, he has been with imec, Leuven, where he has been involved in advanced packaging and interconnect technologies. He is currently a fellow with imec, where he is the Program Director of the 3-D System Integration Program. 